\title{
Cross-Sectional Analysis of Nonhomogeneous Anisotropic Active Slender Structures
}

\author{
Rafael Palacios* and Carlos E. S. Cesnik ${ }^{\dagger}$ \\ University of Michigan, Ann Arbor, Michigan 48109
}

\begin{abstract}
A general formulation for the reduction of the three-dimensional problem of electrothermoelasticity in slender solids to an arbitrarily defined reference line is presented. The dimensional reduction is based on a variationalasymptotic formulation, using the slenderness ratio as small parameter. In the proposed scheme, the coupled linear electroelastic equations are solved at the cross-sectional level using the finite element method. Furthermore, modal components of the displacement field are added to introduce arbitrary deformation shapes into the onedimensional analysis, and arbitrary electric modes are used to define applied electric fields at the cross section. This results in a general definition of a coupled electroelastic stiffness, which can be used in virtually all composite and active beam formulations, as well as in the development of new low-order high-accuracy reduced models for active structures. Finally, the formulation also yields recovery relations for the elastic and electric fields in the original three-dimensional solid, once the one-dimensional problem is solved. The method has been implemented in a computer program (UM/VABS) and numerical results are presented for active anisotropic beam cross sections of simple geometries, which are shown to compare very well with three-dimensional finite element analysis.
\end{abstract}

\begin{tabular}{|c|c|}
\hline & Nomenclature \\
\hline$A$ & $=$ area of the cross section \\
\hline$B$ & $=$ deformed reference frame \\
\hline$b$ & $=$ undeformed reference frame \\
\hline C & $=$ electric capacitance \\
\hline$c_{i j, k l}$ & $=$ material elastic constants (compliances) \\
\hline$D_{i}$ & $=$ electric displacement \\
\hline$d_{i, j k}$ & $=$ inverse piezoelectric constants \\
\hline$E_{i}$ & $=$ electric field \\
\hline$E_{i j}$ & $=$ elastic extensional constants \\
\hline$e_{i, j k}$ & $=$ direct piezoelectric constants \\
\hline$F$ & $=$ column matrix of thermal forces per unit length \\
\hline$G_{i j}$ & $=$ shear modules \\
\hline$g$ & $=$ metric determinant \\
\hline $\mathcal{H}$ & $=$ electric enthalpy per unit length \\
\hline$h$ & $=$ cross-sectional characteristic dimension \\
\hline$K_{i}$ & $=$ curvatures of the deformed reference line \\
\hline$k_{i}$ & $=$ curvatures of undeformed reference line \\
\hline $\begin{array}{lll}n_{l} \\
L\end{array}$ & $=$ typical length along the reference line \\
\hline$P_{i}$ & $=$ components of polarization vector \\
\hline$q$ & $=$ amplitudes of the finite section modes \\
\hline$R$ & $=$ typical radius of the initial curvatures \\
\hline$R_{i}$ & $=$ position of deformed reference line \\
\hline$r_{i}$ & position of undeformed reference line \\
\hline$S$ & electroelastic stiffness matrix \\
\hline$T$ & $\begin{aligned}= & \text { cross section }(\mathrm{C}-\mathrm{S}) \text { finite element method }(\mathrm{FEM}) \\
& \text { nodal values of } \Theta\end{aligned}$ \\
\hline$u$ & $=$ strain energy density \\
\hline$V$ & $=$ electric potential \\
\hline$v$ & $=$ amplitudes of the electric mode shapes \\
\hline
\end{tabular}

Received 25 July 2004; revision received 4 February 2005; accepted for publication 5 July 2005. Copyright (C) 2005 by Rafael Palacios and Carlos E. S. Cesnik. Published by the American Institute of Aeronautics and Astronautics, Inc., with permission. Copies of this paper may be made for personal or internal use, on condition that the copier pay the $\$ 10.00$ per-copy fee to the Copyright Clearance Center, Inc., 222 Rosewood Drive, Danvers, MA 01923; include the code 0001-1452/05 \$10.00 in correspondence with the CCC.

*FXB Fellow, Department of Aerospace Engineering; currently Aerospace Engineer, Structural Dynamics and Aeroelasticity Department, EADS-CASA, Getafe, 28906 Madrid, Spain. Member AIAA.

†Associate Professor, Department of Aerospace Engineering; cesnik@ umich.edu. Associate Fellow AIAA.
$W$

$w_{i}$

$X_{i}$

$x_{i}$

$Y$

$y$

$z$

$\alpha_{i j}$

$\beta_{\bar{y}}, \beta_{\bar{y}}$

$\Gamma_{i j}$

$\gamma_{1 j}$

$\delta$

$\delta_{c}$

$\epsilon$

$\varepsilon_{i j}$

$\Theta$

$\theta$

$\kappa_{i}$

$\mu$

$v_{i j}$

$\Sigma_{i j}$

$\Phi$

$\phi$

$\chi$

$\Psi_{q}^{w}$
$\Psi_{v}^{\phi}$
$\psi_{q}$

$\psi_{v}$

$\Omega$

$\omega$

$\langle\cdot\rangle$

$=\mathrm{C}-\mathrm{S}$ FEM nodal values of $w$

$=$ components of the warping displacement

$=$ particle position in deformed frame

$=$ particle position in undeformed frame

$=\mathrm{C}-\mathrm{S}$ FEM nodal values of $y$

$=$ small-scale state variable at the C-S

$=$ multiscale discrete state variable

$=$ thermal expansion coefficients

$=$ matrix operators for integration by parts

$=$ local strain tensor in the three-dimensional domain

$=$ force strain measures at reference line

$=$ variational operator

$=$ electroelastic coupling parameter

$=$ classical beam strain measures

$=$ dielectric (permittivity) constants

$=$ temperature increment

$=$ material angle

$=$ moment strain measures at reference line

$=$ Lagrange multipliers

$=$ Poisson's ratios

$=$ stress tensor in the three-dimensional domain

$=\mathrm{C}-\mathrm{S}$ FEM nodal values of $\phi$

$=$ electric potential

$=$ long-scale state variable in the one-dimensional domain

$=\mathrm{C}-\mathrm{S}$ FEM nodal values of $\psi_{q}$

$=\mathrm{C}-\mathrm{S}$ FEM nodal values of $\psi_{v}$

$=$ approximating function for finite section warping mode

$=$ applied electric potential mode shapes

$=$ deformed three-dimensional domain

$=$ undeformed three-dimensional domain

$=\mathrm{C}-\mathrm{S}$ integration:

$$
\int_{A} \cdot \sqrt{g} \mathrm{~d} x_{2} \mathrm{~d} x_{3}
$$

Subscripts

$\begin{array}{ll}i j k l & =\text { associated with all three spatial components } \\ s & =\text { symmetric matrix: } 2 A_{p q s}=A_{p q}+A_{q p} \\ \alpha & =\text { associated with } \mathrm{C}-\mathrm{S} \text { components }(2,3)\end{array}$

Superscript

$(\cdot)^{\prime} \quad=$ differentiation with respect to $x_{1} / L$ 


\section{Introduction}

$\mathbf{C}$ ONTROLLING the state of a flexible structure has long been a topic of extensive research, with a good number of technological accomplishments. The ultimate goal is an ideal smart structure that is able to adapt itself through a network of sensors and actuators to the optimum state under varying internal and/or external operating conditions. Wada et al. ${ }^{1}$ introduced a classification of the different approaches for structural control. There, active structures are defined as those in which sensors and actuators are integral parts of the construction with a wider functionality that usually include load-carrying capabilities. Material science provides a wide array of embedded actuators and sensors, with or without load-carrying capabilities, for application in structural control. They can be classified according to the physical mechanism in which they base their active capabilities: piezoelectric, electrostrictive, magnetostrictive, etc. This defines an essentially coupled problem between the elastic field in all load-bearing members and the secondary field(s) (electric, magnetic, thermal) in sensors and actuators. For the case of structures with embedded piezoelectrics, the corresponding governing equations of electrothermoelasticity were derived by Tiersten ${ }^{2}$ and have been applied since then, and with different degrees of simplification, to numerous studies on control of structures with piezoelectric actuators and sensors. ${ }^{3}$ Embedded and surface-mounted piezoactuators are usually modeled considering a prescribed electric field ${ }^{4,5}$ therefore, neglecting the influence on the electric state of the elastic deformation. Analogously, models of sensors usually neglect the influence that the piezoelectrics have in the structural dynamics of the system. ${ }^{6}$ A first attempt to address the coupled electroelastic problem in active structural control is found by Hagood et al., ${ }^{7}$ using a Rayleigh-Ritz approximation. More recently, Ahmad et al. ${ }^{8}$ have developed a finite element solution for the coupled electrothermoelastic problem in beams. Both works show that the solution to the full system can be done with a limited increase in complexity, therefore, avoiding the approximations in the prescribed-field solution.

Most structural subcomponents of mechanical systems can be modeled using only one or two spatial dimensions, that is, as beam or plates. The possibility to do so depends first on the presence of dominant dimensions in their geometry, but also on the characteristic dimensions of the excitation/response under consideration. In this work, the investigation is limited to the analysis of slender structures with embedded piezoelectric actuators and sensors, that is, active beams. The general case of anisotropic material properties both for the passive structural substrate and for the anisotropic piezoelectric actuators and sensors (such as active fiber composites ${ }^{9}$ and macrofiber composites ${ }^{10}$ ) is considered. The focus in this work is in the dimensional reduction of the electrothermoelastic problem in the original three-dimensional domain to a set of properties in a given reference line, which can be initially twisted and curved, and with normal cross sections of arbitrary shape.

With an interest in helicopter blade design, Volovoi et al. ${ }^{11}$ and Jung et al. ${ }^{12}$ have recently reviewed the available modeling methods for anisotropic beams. In general, one can identify two general ways of constructing one-dimensional structural models for heterogeneous and anisotropic flexible slender bodies: by the introduction of ad hoc hypothesis in the displacement field, or by solution through asymptotic expansions. The first approach is the most extensively used, due basically to its effectiveness to obtain a fairly good characterization of very slender structures and with a relatively small degree of anisotropy (conditions that are equivalent to a negligible deviation from the classical isotropic beam theory for the cross-sectional warping). However, the validity of the resulting solutions is hard to guarantee in general situations with nonclassical deformations or with strong elastic couplings. Asymptotic methods, on the other hand, rely on a more complex mathematical foundation but are of general application. In this approach, the slenderness of the (active) structure provides a small parameter to approximate asymptotically the solution to the three-dimensional (electro-) elastic problem, which can be initially posed either in its weak ${ }^{13-15}$ or strong $^{16-19}$ form.

The variational-asymptotic beam cross-sectional (VABS) analysis solution developed by Cesnik and Hodges ${ }^{15}$ provides the basis for the present active beam development. VABS is the application to composite beams of the variational-asymptotic method of Berdichevsky. ${ }^{20}$ In this approach, the cross-sectional warping field corresponding to a set of general-one-dimensional strain measures is solved from the minimization of the asymptotic approximation to the strain energy in the original domain. Asymptotic solutions of composite beams have been pursued in the last decade by Cesnik and his collaborators: Successive contributions have presented the solution to the passive problem for prismatic beams ${ }^{21}$ initially curved and twisted beams, ${ }^{22}$ beams with arbitrary deformation modes ${ }^{23}$ beams with nonperpendicular cross-sectional planes, ${ }^{24}$ and beams with transverse shear effects. ${ }^{25,26}$ Variational-asymptotic solutions for active beams have been also introduced for the thin-walled case ${ }^{5}$ and for the general case of an arbitrary cross section. ${ }^{27}$ However, these active formulations were restricted to the classical beam description (deformation of the reference line defined only by extension, twist, and bending) and for a decoupled form of the electroelastic laws.

In this work, the variational-asymptotic method is applied to the dimensional reduction of the coupled electrothermoelastic equations. The present implementation introduces a modal solution procedure that allows arbitrary definitions of the one-dimensional elastic, thermal, and electric variables. As a result, it defines in a common framework the cross-sectional properties for a wide class of beam models and opens the way for the definition of new refined representations of deformation. The solution process is shown in Fig. 1. When started with an exact description of the three-dimensional electrothermoelastic field and with the use of the condition of slenderness, an approximation to the local state of the system can be determined by the electric enthalpy per unit length of the structure, whereas a one-dimensional problem sets the long-scale behavior of the structure. Without loss of generality, the local deformations can be written in terms of a set of one-dimensional measures of deformation of the reference line and a cross-sectional warping field. The warping acts as a residual error to absorb the difference between

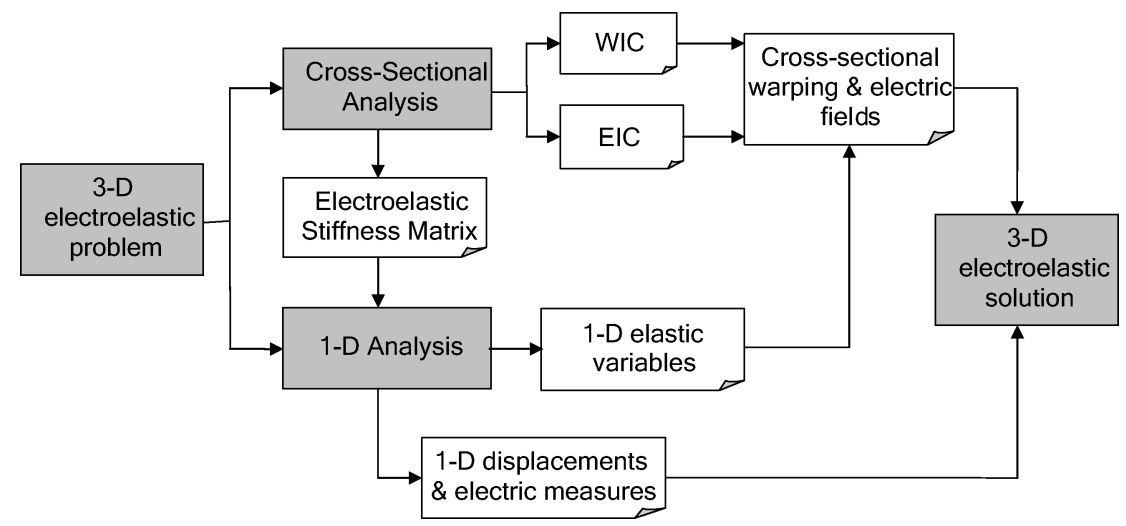

Fig. 1 Asymptotic solution process for three-dimensional electroelasticity in slender structures. 
the one- and three-dimensional representations of the displacement field. In addition to this, the state of the system is also defined by a cross-sectional distribution of electric potential. Both crosssectional fields are not assumed but calculated through an asymptotic expansion on the characteristic cross-sectional length, which is regarded as the small parameter in the problem. They are finally solved as a function of one-dimensional variables by minimization of the cross-sectional electric enthalpy, yielding a set of warping influence coefficients (WIC) and electric influence coefficients (EIC). An independent solution of the one-dimensional problem returns the remaining information to determine the three-dimensional electroelastic field in the original domain. The formulation is based on the assumption of small strain and local rotation, as defined by Danielson and Hodges, ${ }^{28}$ although this does not imply a restriction in the global rotations and displacements of the structure. It also assumes linear electrothermoelastic constitutive relations and that the local electric fields within the active structure can be modeled using linear electrostatics.

\section{Kinematics in the Deformation of Slender Structures}

Consider two configuration states in the deformation process of a slender three-dimensional elastic domain: the undeformed $(\omega)$ and deformed $(\Omega)$ states. A reference line $r$ can be defined along the longitudinal (dominant) direction of the undeformed structure. Its definition is in principle arbitrary, and it does not need to be a straight line. Let $\boldsymbol{r}$ be the position vector of a point in the reference line $r$ and $\boldsymbol{x}$ the position vector of an arbitrary particle in the threedimensional solid. The local Cartesian coordinates are $x_{i}$, such that $x_{\alpha}=0$ corresponds to the reference line. Define now an undeformed reference frame $b$ such as, at the reference line $\boldsymbol{b}_{1}\left(x_{1}\right)$ is tangent to $r$ and the pair $\boldsymbol{b}_{\alpha}\left(x_{1}\right)$ defines the local undeformed cross section. [In this work it will be assumed that the undeformed cross section is normal to the reference line. In some situations (for instance, swept aircraft wings) it may be convenient to define oblique cross sections to simplify the analysis. Popescu et al. ${ }^{24}$ have studied this situation and introduced transformation rules from oblique to normal cross sections.] The position vector $\boldsymbol{x}$ of a given point in the undeformed structure can be written as

$$
\boldsymbol{x}\left(x_{1}, x_{2}, x_{3}\right)=\boldsymbol{r}\left(x_{1}\right)+x_{\alpha} \boldsymbol{b}_{\alpha}\left(x_{1}\right)
$$

To define the deformed configuration, let $\boldsymbol{X}$ be the position vector after deformation of a particle initially at $\boldsymbol{x}$ and $\boldsymbol{R}$ the averaged value of $\boldsymbol{X}$ on the cross sections of $r$,

$$
\boldsymbol{R}\left(x_{1}\right)=\langle\boldsymbol{X}\rangle / A\left(x_{1}\right)
$$

with $A\left(x_{1}\right)=\langle 1\rangle$. The position vector $\boldsymbol{R}$ then defines the deformed reference line $R$. An intrinsic orthogonal reference frame is defined ${ }^{28}$ at each point along $R, \boldsymbol{B}_{i}\left(x_{1}\right)$, the deformed reference frame $B$. The definition of $B$ is not unique, and two different major conventions can be adopted, corresponding to generalizations of the Euler-Bernoulli and Timoshenko models for isotropic homogeneous beams. In the first case, the deformed frame is defined with $\boldsymbol{B}_{1}$ tangent to $R$. In the second, the deformed frame is defined such as the pair $\boldsymbol{B}_{\alpha}$ is in the plane obtained by the deformation of $\boldsymbol{b}_{\alpha}$. This work takes the first definition, which corresponds to Frenet's description of a space curve and yields a natural solution to the asymptotic expansions. Once $\boldsymbol{B}_{1}$ is fixed, one last condition will determine the orientation of the vectors $\boldsymbol{B}_{\alpha}$ within their plane. It comes from imposing that the average in-plane rotation in the deformed frame is zero, as

$$
\left\langle x_{3}(\boldsymbol{X}-\boldsymbol{R}) \cdot \boldsymbol{B}_{2}-x_{2}(\boldsymbol{X}-\boldsymbol{R}) \cdot \boldsymbol{B}_{3}\right\rangle=0
$$

Note that this condition is actually defining the torsion angle at the cross section as the in-plane rotation between the undeformed and the deformed reference frames. As a result, the position vector in the deformed state of the material point defined in Eq. (1) can be written, without loss of generality, as

$$
\boldsymbol{X}\left(x_{1}, x_{2}, x_{3}\right)=\boldsymbol{R}\left(x_{1}\right)+x_{\alpha} \boldsymbol{B}_{\alpha}\left(x_{1}\right)+\boldsymbol{w}\left(x_{1}, x_{2}, x_{3}\right)
$$

A new magnitude, the warping field $\boldsymbol{w}$ measures the deformation of the cross section from its original shape after rigid-body translations and rotations have been removed. In general, the slenderness of the structure implies that the warping is small, and this will be the basis for the asymptotic dimensional reduction in the next section. To get a unique description of the deformation, the warping field must satisfy

$$
\left\langle\boldsymbol{w} \cdot \boldsymbol{B}_{i}\right\rangle=0, \quad\left\langle x_{3}\left(\boldsymbol{w} \cdot \boldsymbol{B}_{2}\right)-x_{2}\left(\boldsymbol{w} \cdot \boldsymbol{B}_{3}\right)\right\rangle=0
$$

The first condition comes from imposing Eq. (2), whereas the second condition replaces Eq. (3), as can be easily checked. The warping is now decomposed using a Ritz approximation into two components: 1) an assumed field, given by a set of cross-sectional deformation approximating functions, and 2) a residual warping, which completes the description of the three-dimensional deformation:

$$
\boldsymbol{w}\left(x_{1}, x_{2}, x_{3}\right)=\sum_{m=1}^{N_{q}} q_{m}\left(x_{1}\right) \boldsymbol{\psi}_{q m}\left(x_{2}, x_{3}\right)+\overline{\boldsymbol{w}}\left(x_{1}, x_{2}, x_{3}\right)
$$

The distributions of cross-sectional displacements $\left\{\psi_{q m}\right\}$ are called herein finite section deformation modes and can be regarded as orthogonal elements of the functional space of all possible crosssectional deformations that satisfy Eq. (5). These displacement modes can have components in and out of the cross-sectional plane and are selected a priori. Here $\left\{q_{m}\right\}$ are the corresponding amplitudes that will define additional variables in the reduced problem on the reference line. The orthogonality conditions between the modes are

$$
\left\langle\left(\boldsymbol{\psi}_{q m} \cdot \boldsymbol{B}_{i}\right)\left(\boldsymbol{\psi}_{q n} \cdot \boldsymbol{B}_{i}\right)\right\rangle=\delta_{m n}
$$

In general, the kinematics of a deformable curve is well represented by its four intrinsic variables ${ }^{28}$ : three curvatures (torsion and bending in two directions) and the extensional strain. [Note that a shear component can be defined in the deformation of the threedimensional body, but not between the undeformed and deformed reference frames. This does not imply that shear is neglected in the present formulation, but that it must be then considered as part of the warping field in Eq. (4). An illustration of this is included in the first sample case later in this work.] They are written here as a $4 \times 1$ vector of classical beam strains:

$$
\epsilon\left(x_{1}\right)=\left\{\gamma_{11}, h \kappa_{1}, h \kappa_{2}, h \kappa_{3}\right\}
$$

where $h$ is the characteristic length of the cross section and it is used to get dimensionless curvatures. In addition to this, $\gamma_{11}$ is the axial force strain measure and $\kappa_{i}$ are the three moment strains measures, defined as the difference between the components in the curvature vectors of the deformed $(\boldsymbol{K})$ and undeformed $(\boldsymbol{k})$ reference line in their respective basis,

$$
\kappa_{i}=\boldsymbol{K} \cdot \boldsymbol{B}_{i}-\boldsymbol{k} \cdot \boldsymbol{b}_{i}
$$

Following the work of Danielson and Hodges ${ }^{28}$ the deformation of the three-dimensional elastic solid is characterized by the Jaumann-Biot-Cauchy strain tensor. Its independent components are written in the form of a column matrix $\Gamma^{T}=\left\{\Gamma_{11}, 2 \Gamma_{12}, 2 \Gamma_{13}\right.$, $\left.\Gamma_{22}, 2 \Gamma_{23}, \Gamma_{33}\right\}$. For the displacement field of Eq. (4), and under the assumption of small strain and local rotation, the strains are written as $^{15}$

$$
\Gamma(\boldsymbol{x})=\Gamma_{\epsilon} \epsilon\left(x_{1}\right)+\Gamma_{h} \hat{w}(\boldsymbol{x})+(h / R) \Gamma_{R} \hat{w}(\boldsymbol{x})+(h / L) \Gamma_{l} \hat{w}^{\prime}
$$

where $\hat{w}$ are the components of the nondimensional warping vector $\boldsymbol{w} / h$ in the deformed reference frame $B$ and the matrix operators in Eq. (10) are defined as done by Cesnik and Hodges, ${ }^{15}$ 


$$
\begin{aligned}
& \Gamma_{\epsilon}^{T}=\frac{1}{\sqrt{g}}\left[\begin{array}{cccccc}
1 & 0 & 0 & 0 & 0 & 0 \\
0 & -\hat{x}_{3} & \hat{x}_{2} & 0 & 0 & 0 \\
\hat{x}_{3} & 0 & 0 & 0 & 0 & 0 \\
-\hat{x}_{2} & 0 & 0 & 0 & 0 & 0
\end{array}\right] \\
& \Gamma_{h}^{T}=\left[\begin{array}{cccccc}
0 & \frac{\partial}{\partial \hat{x}_{2}} & \frac{\partial}{\partial \hat{x}_{3}} & 0 & 0 & 0 \\
0 & 0 & 0 & \frac{\partial}{\partial \hat{x}_{2}} & \frac{\partial}{\partial \hat{x}_{3}} & 0 \\
0 & 0 & 0 & 0 & \frac{\partial}{\partial \hat{x}_{2}} & \frac{\partial}{\partial \hat{x}_{3}}
\end{array}\right] \\
& \Gamma_{R}=\frac{1}{\sqrt{g}}\left[\begin{array}{ccc}
0 & -\hat{k}_{3} & \hat{k}_{2} \\
\hat{k}_{3} & 0 & -\hat{k}_{1} \\
-\hat{k}_{2} & \hat{k}_{1} & 0 \\
& 0_{3} &
\end{array}\right]+\frac{1}{\sqrt{g}} \hat{k}_{1}\left(\hat{x}_{3} \frac{\partial}{\partial \hat{x}_{2}}-\hat{x}_{2} \frac{\partial}{\partial \hat{x}_{3}}\right)\left[\begin{array}{l}
I_{3} \\
0_{3}
\end{array}\right] \\
& \Gamma_{l}=\frac{1}{\sqrt{g}}\left[\begin{array}{l}
I_{3} \\
0_{3}
\end{array}\right]
\end{aligned}
$$

In these expressions, $\hat{x}_{\alpha}=x_{\alpha} / h$ and $\hat{k}_{i}=R k_{i}$ are the nondimensional cross-sectional coordinates and the initial curvatures of the reference line, respectively, with $1 / R=\max \left(\left\|k_{i}\right\|\right)$. The metric determinant $g$ is a function of the initial bending curvatures of the reference line:

$$
\sqrt{g}=1-(h / R)\left(\hat{x}_{2} \hat{k}_{3}+\hat{x}_{3} \hat{k}_{2}\right)
$$

\section{Three-Dimensional Static Electroelastic Equilibrium Conditions}

Consider a dielectric continuum in a slender domain, $\Omega=$ $[0, l] \times A$, having electroelastic properties, that is, it is electrically polarizable and elastically deformable. This section develops the static equilibrium conditions on this domain using the definitions in Ref. 29. Let $\boldsymbol{P}(\boldsymbol{x})$ be the polarization vector at a given location and assume an electric loading that generates an electric field $\boldsymbol{E}(\boldsymbol{x})$ in the direction of polarization. $\boldsymbol{D}(\boldsymbol{x})$ is the local electric displacement from the interaction between fields $\boldsymbol{E}$ and $\boldsymbol{P}$. When linear electrostatics are assumed, the internal electric energy density is then given by $u^{e}=\frac{1}{2} \boldsymbol{D}(\boldsymbol{x}) \cdot \boldsymbol{E}(\boldsymbol{x})$. The second contribution to the energy state comes from the elastic deformation. The local elastic state is defined by the values of the strain and stress tensors, $\Gamma(\boldsymbol{x})$ and $\Sigma(\boldsymbol{x})$, respectively. When linear elasticity is assumed, and if linear thermal effects are included (local dimensionless temperature increment $\Theta$ ), the strain energy density is given by $u^{s}=\frac{1}{2} \boldsymbol{\Sigma}(\boldsymbol{x}):[\boldsymbol{\Gamma}(\boldsymbol{x})-\boldsymbol{\alpha}(\boldsymbol{x}) \cdot \Theta(\boldsymbol{x})]$. Static equilibrium in $\Omega$ is determined by the variational problem ${ }^{29}$

$$
\delta \int_{\Omega}\left(u^{s}+u^{e}-\boldsymbol{E} \cdot \boldsymbol{D}\right) \mathrm{d} V=\delta W_{\Omega}^{s}+\delta W_{\partial \Omega}^{s}
$$

where the pair of terms in the right-hand side of Eq. (13) corresponds to the work of the external volume and surface forces on the domain $\Omega$ and its boundary $\partial \Omega$, respectively.

Consider now the cross section at a certain position $x_{1}$ in the reference line. Figure 2 shows a schematic representation of a general section of arbitrary shape that includes an elastic material with embedded piezoelectric actuators and sensors. If the integral in Eq. (13) is first evaluated at each cross section, it defines the electric enthalpy per unit length of the reference line, $\mathcal{H}$ (or cross-sectional enthalpy) as

$$
\mathcal{H}=\left\langle u^{s}+u^{e}-D^{T} E\right\rangle
$$

Vector magnitudes are expressed as the column matrices of their components in the deformed frame $B$. The final form of the crosssectional enthalpy is constructed by assuming that the material properties are given by linear electroelastic constitutive relations. It is

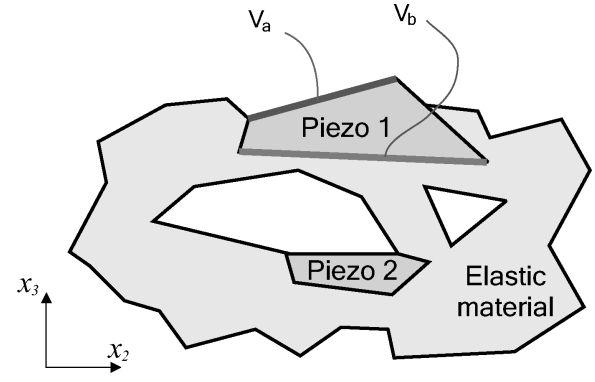

Fig. 2 Cross section with embedded actuator (piezo 1, with voltages $V_{a}$ and $V_{b}$ ) and sensor (piezo 2).

also assumed that the material coefficients are independent of the magnitude and frequency of the applied mechanical stresses and electric fields:

$$
\left\{\begin{array}{l}
\Sigma \\
D
\end{array}\right\}=\left[\begin{array}{cc}
c^{E} & -e^{T} \\
e & \varepsilon^{\Gamma}
\end{array}\right] \cdot\left\{\begin{array}{c}
\Gamma^{m} \\
E
\end{array}\right\}
$$

The superscript $E$ stands for values at constant electric field and $\Gamma$ for values at constant strain. The coupling between the electric and the elastic fields is induced by the matrix of direct piezoelectric constants $e$, which are referred to the local poling direction. For this constitutive relations and if the electric field is written in terms of the electric potential, $E=-\nabla \phi$, the cross-sectional electric enthalpy of Eq. (14) is defined as a functional of the three-dimensional strain, temperature, and electric potential fields:

$$
\mathcal{H}=\frac{1}{2}\left\langle\Gamma^{T} c^{E} \Gamma\right\rangle-\frac{1}{2}\left\langle\nabla \phi^{T} \varepsilon^{\Gamma} \nabla \phi\right\rangle+\left\langle\Gamma^{T} e^{T} \nabla \phi\right\rangle-\left\langle\Gamma^{T} c^{E} \alpha \Theta\right\rangle
$$

\section{Small Parameters and Dimensional Reduction}

The first step in the dimensional reduction is the identification of the actual characteristic lengths involved in the process of deformation of the slender structure. They are 1) the cross-sectional characteristic dimension $h, 2)$ the characteristic wavelength of the response along the longitudinal dimension $L$ (depending both on the longitudinal rate of change of the geometrical and material properties and on the typical wavelength on the excitation), and 3) the characteristic length of the initial radius of curvature and twist, $R$. In what follows, it is assumed that the characteristic dimension of the cross section, $h$, is small compared to the longitudinal dimension and the typical radius of curvature. Moreover, it will be assumed that $L$ and $R$ are of the same order, that is, $h \ll L \approx R$. Therefore, the single small parameter $\hat{h}$ defined as

$$
\hat{h}=h / L \approx h / R \ll 1
$$

is introduced. The classical beam strains $\epsilon$ are an additional small parameter in the problem. Their effect has already been considered when linearizing the three-dimensional strain field. To obtain oneparameter asymptotic expansions, it is assumed that $\max \|\epsilon\|$ and $\hat{h}$ are of the same order and the following scaling is defined on $\epsilon$ :

$$
\hat{\epsilon}=\hat{h}^{-1} \epsilon \sim 1
$$

Analogously, the thermal strains are assumed to be of the same order as the mechanical strains, $\max \|\alpha \Theta\| \sim \max \|\epsilon\| \sim \hat{h}$, and the cross-sectional temperature field is correspondingly rescaled as

$$
\hat{\Theta}=\hat{h}^{-1} \Theta \sim 1
$$

The small parameter $\hat{h}$ defines two different scales in the variational problem (13): a long-scale problem, defined by the deformation of the reference line, and a small-scale problem, given by the local (warping) deformations at the cross section. When the description of the kinematics introduced earlier is used, it is possible to identify long- and small-scale contributions to the cross-sectional electric enthalpy (16). From Eqs. (6) and (10), the column matrix with the components of the local strain tensor $\Gamma$ is expressed in terms of the 
value of the classical beam strain measures, $\hat{\epsilon}$, the amplitudes of the finite-section deformation modes, $q$, and the residual warping field, $\bar{w}$. In a similar manner, the electric potential distribution at each cross section can be split into two components: $\phi=\psi_{v} v+\bar{\phi}$. The first term is the applied electric potential ( $V_{a}$ and $V_{b}$ in Fig. 2), defined through a set of independent (and given) electric modes $\psi_{v}\left(x_{2}, x_{3}\right)$ and their dimensionless amplitudes, $v\left(x_{1}\right)$; the second component is the induced potential in the interior of the dielectric domain, $\bar{\phi}\left(x_{2}, x_{3}\right)$. As a result, one can write the cross-sectional electric enthalpy as

$$
\mathcal{H}=\mathcal{H}\left(\chi, \bar{y}, \bar{y}^{\prime}, \Theta\right)
$$

where, $\chi$ and $\bar{y}$ are defined as the long- and small-scale state variables, defined as

$$
\chi=\left\{\hat{\epsilon}, v, v^{\prime}, q, q^{\prime}\right\}, \quad \bar{y}=\{\bar{w}, \bar{\phi}\}
$$

One can approximately find the solution to the variational problem (13) by assuming that the long- and small-scale variables can be solved independently. ${ }^{13}$ The small-scale problem is defined by the minimization of the electric enthalpy of Eq. (20) in the small-scale variable $\bar{y}$ for prescribed values of the long-scale variable $\chi=\chi_{0}$ and the temperature field $\Theta=\Theta_{0}$. The solution in this minimization process has to satisfy the conditions on the cross-sectional displacement field introduced by Eqs. (5) and (7). As a result, the sectional problem is given as a constrained minimization problem, which in symbolic form is written as

$$
\delta \mathcal{H}\left(\bar{y}, \bar{y}^{\prime} ; \chi_{0}, \Theta_{0}\right)=0
$$

subject to $C\left(\bar{y}, \chi_{0}\right)=0$, where $C$ represents the orthogonality constraints on the warping field. In summary, the cross-sectional enthalpy (16) is expressed as a functional in the given (onedimensional) long-scale state variable $\chi$, the given (cross-sectional) distribution of temperatures, $\Theta$, the unknown (cross-sectional) small-scale state variable $\bar{y}$, and its spatial derivatives along the reference line, $\bar{y}^{\prime}$. The constrained minimization problem (22) is then defined in the cross section and yields a linear relation between the small-scale variable $\bar{y}$ and the given values of the long-scale variable and temperature field, $\chi=\chi_{0}$ and $\Theta=\Theta_{0}$. The pair $\chi_{0}$ and $\Theta_{0}$ is computed from the dynamics of the long-scale problem (which is not considered in this work) and is assumed to be known in the cross-sectional analysis. Note that in this formulation the long-scale state variable $\chi$ includes measures of nonclassical cross-sectional $q$ and longitudinal $q^{\prime}$ strains, as well as cross-sectional $v$ and longitudinal $v^{\prime}$ electric fields. In the small-scale problem, the cross section is defined by the orthogonal coordinates $x_{\alpha}$, whereas the coordinate along the reference curve, $x_{1}$, can be considered locally normal to the cross-sectional plane.

\section{Finite Element Formulation of the Cross-Sectional Problem}

For the general case of anisotropic material and arbitrary cross sections, the electroelastic compliance matrices in Eq. (15) are fully populated and vary in the cross section. The variational problem (22) can only be solved through a numerical procedure, and the finite element method (FEM) is used here. As it was seen, three different vector and scalar fields are defined in the cross section: displacements (warping), electric potential, and temperature. Their discretization in finite elements is

$$
\begin{aligned}
& \hat{w}\left(x_{1}, x_{2}, x_{3}\right)=N_{w}\left(x_{2}, x_{3}\right) W\left(x_{1}\right) \\
& \phi\left(x_{1}, x_{2}, x_{3}\right)=N_{\phi}\left(x_{2}, x_{3}\right) \Phi\left(x_{1}\right) \\
& \Theta\left(x_{1}, x_{2}, x_{3}\right)=N_{T}\left(x_{2}, x_{3}\right) T\left(x_{1}\right)
\end{aligned}
$$

If there are $n$ grid nodes in the cross section, $W$ is a column matrix of dimension $3 n$ corresponding to the three components of the nondimensional warping field at each node, whereas $\Phi$ and $T$ are column matrices of dimension $n$ with the nodal values of the electric potential and temperature fields, respectively. The matrices $N_{W}, N_{\Phi}$,

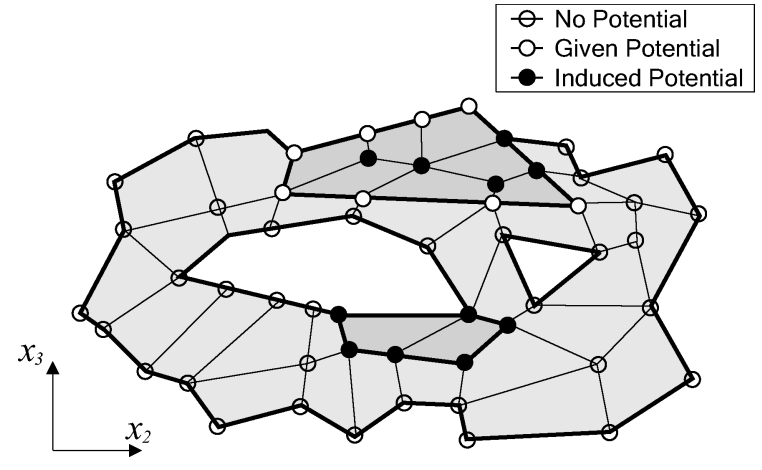

Fig. 3 Distribution of electric potential in finite element discretization of cross section of Fig. 2.

and $N_{T}$ define the shape functions corresponding to each crosssectional field. For the nodal distribution of electric potential, the cross-sectional domain is split in the general case into three subsets, as shown in Fig. 3, corresponding to interior nodes in the region of purely elastic material (null potential), the regions within the actuators with prescribed values of the potential (applied potential), and the interior nodes in the piezoelectric inclusions (induced potential). If the nodes were appropriately reordered, the discrete form of the electric potential would be written as

$$
\Phi=\left\{\begin{array}{c}
\bar{\Phi} \\
0 \\
0
\end{array}\right\}+\left\{\begin{array}{c}
0 \\
\Psi_{v}^{\phi} \\
0
\end{array}\right\} v
$$

where $\Psi_{v}^{\phi}$ is the matrix of independent electric excitation modes that is defined in the nodes with given potential and $\bar{\Phi}$ is the vector of induced electric potential at nodes.

For the displacement field $W$, the domain of discretization is the complete cross section. The discrete (matrix) form of the modal expansion defined in Eq. (6) is used now to capture higher-order components of the deformation, $W=\Psi_{q}^{w} q+\bar{W}$. The finite element representation allows numerical integration of the coefficients of the cross-sectional enthalpy (20), which can be written in discrete form as a bilinear operator:

$$
\mathcal{H}(z ; \hat{T})=\frac{1}{2} z^{T}\left(A_{0}+\hat{h} A_{1}+\hat{h}^{2} A_{2}\right) z+z^{T}\left(\hat{h} b_{1}+\hat{h}^{2} b_{2}\right) \hat{T}
$$

where the different constant submatrices are given in the Appendix. The new multiscale state variable $z$ is

$$
z=\left\{\begin{array}{c}
\chi \\
\bar{Y} \\
\bar{Y}^{\prime}
\end{array}\right\}, \quad \text { with } \quad \bar{Y}=\left\{\begin{array}{c}
\bar{W} \\
\bar{\Phi}
\end{array}\right\}
$$

Therefore, the state variable $z$ is the combination of the long-scale state variable $\chi$, the discrete small-scale state variable $\bar{Y}$, and its longitudinal derivative. Note that, whereas $\bar{W}$ is defined over the whole domain of the cross section, $\bar{\Phi}$ is only at the interior nodes of the dielectric regions. Because of this, it is interesting to rewrite the first term in Eq. (25) as

$$
z^{T} A_{0} z=\left\{\begin{array}{c}
\bar{Y}+\Psi_{q}^{\bar{y}} q \\
\Psi_{v}^{\phi} v
\end{array}\right\}^{T}\left[\begin{array}{cc}
E_{\overline{y y}} & E_{\bar{y} \phi} \\
E_{\phi \bar{y}} & E_{\phi \phi}
\end{array}\right]\left\{\begin{array}{c}
\bar{Y}+\Psi_{q}^{\bar{y}} q \\
\Psi_{v}^{\phi} v
\end{array}\right\}
$$

where the matrix $\Psi_{q}^{\bar{y}}$ is defined by adding zeros to the columns of $\Psi_{q}^{w}$ up to the dimension of $\bar{Y}$. Matrices $E_{\overline{y y}}, E_{\bar{y} \phi}=E_{\phi \bar{y}}^{T}$, and $E_{\phi \phi}$ are constant matrices obtained from the cross-sectional integration.

It was seen earlier that, for a unique definition of the warping field, this must satisfy conditions (5). From the point of view of a crosssectional analysis, those conditions are equivalent ${ }^{15}$ to removing from the warping its rigid-body components (three translations and the in-plane rotation). Those conditions are to be expressed now in 
discrete form. If $\Psi_{\epsilon}^{w}$ is a $3 n \times 4$ matrix whose columns are those rigid-body motions, the set of conditions (5) can be formulated as orthogonal constraints,

$$
\bar{W}^{T} H_{w w} \Psi_{\epsilon}^{w}=0
$$

The notation $H_{x y}$ refers to the matrix operator for the cross-sectional integration of the product of two vector fields, $X$ and $Y$. For finite element discretizations as in Eq. (23), it is $H_{x y}=\left\langle N_{x}^{T} N_{y}\right\rangle$. It can be proved that the rigid-body motions in Eq. (28) (written as $\Psi_{\epsilon}^{\bar{y}}$, which is again obtained by expanding with zeros $\Psi_{\epsilon}^{w}$ up to the size of $\bar{Y}$ ) span the kernel of $E_{\overline{y y}}$ in Eq. (27). An equivalent definition for $\Psi_{\epsilon}^{\bar{y}}$ is then

$$
E_{\overline{y y}} \Psi_{\epsilon}^{\bar{y}}=0, \quad \text { with } \quad\left(\Psi_{\epsilon}^{\bar{y}}\right)^{T} H_{\overline{y y}} \Psi_{\epsilon}^{\bar{y}}=I
$$

The last equation is just a normalization condition on $\Psi_{\epsilon}^{\bar{y}}$. For the finite section deformation modes, the uniqueness of the displacement field is obtained by the orthogonality conditions (7), which in discrete form are written as

$$
\begin{aligned}
\left(\Psi_{q}^{\bar{y}}\right)^{T} H_{\overline{y y}} \Psi_{q}^{\bar{y}} & =I, & & \left(\Psi_{q}^{\bar{y}}\right)^{T} H_{\overline{y y}} \Psi_{\epsilon}^{\bar{y}}=0 \\
\bar{Y}^{T} H_{\overline{y y}} \Psi_{q}^{\bar{y}} & =0, & & \bar{Y}^{T} H_{\overline{y y}} \Psi_{\epsilon}^{\bar{y}}=0
\end{aligned}
$$

\section{Asymptotic Solution for the Warping and Electric Potential}

The solution of problem (22) is sought using an asymptotic expansion in the small parameter $\hat{h}$. The expansion up to order $N$ of the unknown warping and electric potential fields is

$$
\bar{Y}=\sum_{n=0}^{N} \hat{h}^{n} \bar{Y}_{n}+\mathcal{O}\left(\hat{h}^{N+1}\right)
$$

An alternative form of this expansion can be introduced in terms of the variable $z$ defined in Eq. (26),

$$
\begin{gathered}
z=\sum_{n=0}^{N} \hat{h}^{n} z_{n}+\mathcal{O}\left(\hat{h}^{N+1}\right) \\
z_{0}=\left\{\begin{array}{c}
\chi \\
\bar{Y}_{0} \\
\bar{Y}_{0}^{\prime}
\end{array}\right\}, \quad z_{n}=\left\{\begin{array}{c}
0 \\
\bar{Y}_{n} \\
\bar{Y}_{n}^{\prime}
\end{array}\right\}, \quad \text { for } \quad n>0
\end{gathered}
$$

As it will be clear later, the introduction of this last expansion brings a considerably simplification into the solution process. Note that $z$ includes both the one-dimensional state variable $\chi\left(x_{1}\right)$ (the given data) and the nodal values of the finite element discretization of the unknown cross-sectional fields, $\bar{Y}$. Substitution of Eq. (32) in Eq. (25) defines an asymptotic expansion of the cross-sectional enthalpy:

$$
\mathcal{H}=\sum_{n=0}^{N} \hat{h}^{n} \mathcal{H}_{n}+\mathcal{O}\left(\hat{h}^{N+1}\right)
$$

The terms $\bar{Y}_{n}$ are recursively obtained through the sequential minimization of the components of the expansion (33). Thanks to the quadratic nature of the definition of the enthalpy (25), it can be shown along the solution process that the $2 \mathrm{~N}$ th-order expansion of $\mathcal{H}$ depends only on the $N$ expansion on $z$. The asymptotic expansion (33) can be then rewritten as

$$
\begin{aligned}
\mathcal{H}= & \mathcal{H}_{0}\left(z_{0}\right)+\sum_{n=1}^{N}\left(\hat{h}^{2 n-1} \mathcal{H}_{2 n-1}\left(z_{0}, \ldots, z_{n} ; \hat{T}\right)\right. \\
& \left.+\hat{h}^{2 n} \mathcal{H}_{2 n}\left(z_{0}, \ldots, z_{n} ; \hat{T}\right)\right)+\mathcal{O}\left(\hat{h}^{2 N+1}\right)
\end{aligned}
$$

Furthermore, the leading terms in the even components of this expansion $\mathcal{H}_{2 n}$ are quadratic terms in $z_{n}$, and their minimization yields the $n$ th-order solution for the warping and electric field. This is done next.

\section{Zero-Order Solution}

The zero-order components of the unknown warping and potential cross-sectional fields, $\bar{Y}_{0}$, are obtained from the minimization of $\mathcal{H}_{0}$ with constraints (28) and (30). The zero-order enthalpy is

$$
\mathcal{H}_{0}=\frac{1}{2} z_{0}^{T} A_{0} z_{0}
$$

When Eq. (27) is used and the Lagrange multipliers that enforce the orthogonality constraints $\mu_{0}$ are introduced, the Euler equation for the problem can be written as

$$
E_{\overline{y y}}\left(\bar{Y}_{0}+\Psi_{q}^{\bar{y}} q\right)+E_{\bar{y} v} \Psi_{v}^{\phi} v=H_{\overline{y y}} \Psi_{\epsilon}^{\bar{y}} \mu_{0}^{\epsilon}+H_{\overline{y y}} \Psi_{q}^{\bar{y}} \mu_{0}^{q}
$$

Premultiplying successively Eq. (36) by the transpose of the kernel $\Psi_{\epsilon}^{\bar{y}}$ and by the assumed modes $\Psi_{q}^{y}$, and using the orthogonality conditions, one gets the following relations:

$$
\mu_{0}^{\epsilon}=0, \quad \mu_{0}^{q}=\mu_{0 q} q+\mu_{0 v} v
$$

As a result, the solution to this linear system can be written as

$$
\bar{Y}_{0}=\bar{Y}_{0 q} q+\bar{Y}_{0 v} v
$$

and the linear dependence of $\bar{Y}_{0}$ with $q$ and $v$ needs to be found. The dependence on amplitudes of the finite section deformation modes $q$ is obtained as follows. First, differentiation of Eq. (36) in $q$ yields

$$
E_{\overline{y y}}\left(\bar{Y}_{0 q}+\Psi_{q}^{\bar{y}}\right)=H_{\overline{y y}} \Psi_{q}^{\bar{y}} \mu_{0 q}
$$

where $E_{\overline{y y}}$ is singular in the displacements. When conditions Eq. (30) are used, this equation can be rewritten as

$$
E_{\overline{y y}}\left(\bar{Y}_{0 q}+\Psi_{q}^{\bar{y}}\right)=\Lambda_{0} H_{\overline{y y}} \Psi_{q}^{\bar{y}} \mu_{0 q}, \quad \text { with } \quad \Lambda_{0}=I-H_{\overline{y y}} \Psi_{\epsilon}^{\bar{y}}\left(\Psi_{\epsilon}^{\bar{y}}\right)^{T}
$$

The right-hand side in this equation is orthogonal to the kernel of $E_{\overline{y y}}$, that is, $\Psi_{\epsilon}^{\bar{y}}$. Therefore, the modified system has a unique solution, and the matrix $E_{0}=\left(\Lambda_{0}\right)^{-1} E_{\overline{y y}}$ is defined for which a pseudoinverse can be obtained in the solution. (Abusing notation, it will be written as $E_{0}^{-1}$.) The Lagrange multipliers $\mu_{0 q}$ are computed after imposing the corresponding orthogonality conditions from constraits (30):

$$
\mu_{0 q}=\left[\left(\Psi_{q}^{\bar{y}}\right)^{T} H_{\overline{y y}} E_{0}^{-1} H_{\overline{y y}} \Psi_{q}^{\bar{y}}\right]^{-1}
$$

Finally, substitution of Eq. (41) into Eq. (39) gives the zero-order dependency on the finite section modes of the asymptotic expansion of the unknown warping and potential cross-sectional fields:

$$
\bar{Y}_{0 q}=E_{0}^{-1} H_{\overline{y y}} \Psi_{q}^{\bar{y}} \mu_{0 q}-\Psi_{q}^{\bar{y}}
$$

Similarly, the dependence on the amplitudes $v$ of the electric modes comes from

$$
E_{\overline{y y}} \bar{Y}_{0 v}+E_{\bar{y} v} \Psi_{v}^{\phi}=H_{\overline{y y}} \Psi_{q}^{\bar{y}} \mu_{0 v}
$$

As before, the singularity of $E_{\overline{y y}}$ in the displacements domain is removed by factorizing the right-hand side by $\Lambda_{0}$ as in Eq. (40). The Lagrange multipliers are equally computed from orthogonality conditions (30),

$$
\mu_{0 v}=\mu_{0 q}^{T}\left(\Psi_{q}^{\bar{y}}\right)^{T} H_{\overline{y y}} E_{0}^{-1} E_{\bar{y} v} \Psi_{v}^{\phi}
$$

and the zero-order dependency on the applied electric modes is

$$
\bar{Y}_{0 v}=E_{0}^{-1} H_{\overline{y y}} \Psi_{q}^{\bar{y}} \mu_{0 v}-E_{0}^{-1} E_{\bar{y} v} \Psi_{v}^{\bar{y}}
$$

The first term in Eq. (45) comes from imposing orthogonality of the unknown warping and the assumed modes. The second is the solution to a boundary problem for the electric potential in the cross section. 


\section{First-Order Solution}

Once $\bar{Y}_{0}$ is known, the first-order term in the expansion, $\bar{Y}_{1}$, can be computed from the next even term in the expansion of the enthalpy (34), that is, $\mathcal{H}_{2}$, given by

$$
\mathcal{H}_{2}=\left\{\begin{array}{ll}
z_{0}^{T} & z_{1}^{T}
\end{array}\right\} \cdot\left(\frac{1}{2}\left[\begin{array}{ll}
A_{2} & A_{1} \\
A_{1} & A_{0}
\end{array}\right] \cdot\left\{\begin{array}{l}
z_{0} \\
z_{1}
\end{array}\right\}+\left\{\begin{array}{l}
b_{2} \\
b_{1}
\end{array}\right\} \hat{T}\right)
$$

As before, the unknown fields are obtained by minimization of $\mathcal{H}_{2}$ with orthogonality constraints (28) and (30). If $\mu_{1}$ denotes the Lagrange multipliers that enforce this constraint, the Euler equation to compute $\bar{Y}_{1}$ is

$$
E_{\overline{y y}} \bar{Y}_{1}+\beta_{\bar{y}}\left(A_{1} z_{0}+b_{1} \hat{T}\right)-\beta_{\bar{y}^{\prime}} A_{1} z_{0}^{\prime}=H_{\overline{y y}} \Psi_{\epsilon}^{\bar{y}} \boldsymbol{\mu}_{1}^{\epsilon}+H_{\overline{y y}} \Psi_{q}^{\bar{y}} \boldsymbol{\mu}_{1}^{q}
$$

where $\beta_{\bar{y}}$ and $\beta_{\bar{y}^{\prime}}$ are rectangular matrices of dimension size $(\bar{Y}) \times(z)$ and are defined in the integration by parts to eliminate terms in $\bar{Y}_{1}^{\prime}$. Their values are $\beta_{\bar{y}}=\left[\begin{array}{lll}0 & I & 0\end{array}\right]$ and $\beta_{\bar{y}^{\prime}}=\left[\begin{array}{lll}0 & 0 & I\end{array}\right]$. Vectors $\mu_{1}^{\epsilon}$ and $\mu_{1}^{q}$ are obtained by premultiplying this expression with the transposes of $\Psi_{\epsilon}^{\bar{y}}$ and $\Psi_{q}^{\vec{y}}+\bar{Y}_{0 q}$, respectively, and then applying the orthogonality conditions. We introduce now the following matrix definitions:

$$
\begin{aligned}
\Lambda_{u} & =\left[I-H_{\overline{y y}} \Psi_{\epsilon}^{\bar{y}}\left(\Psi_{\epsilon}^{\bar{y}}\right)^{T}-H_{\overline{y y}} \Psi_{q}^{\bar{y}}\left(\Psi_{q}^{\bar{y}}+\bar{Y}_{0 q}\right)^{T}\right] \\
& =\left(I-H_{\overline{y y}} \Psi_{\epsilon} \Psi_{\epsilon}^{T}\right) \cdot\left(I-H_{\overline{y y}} \Psi_{q}^{\bar{y}}\left(\Psi_{q}^{\bar{y}}+\bar{Y}_{0 q}\right)^{T}\right] \\
E_{u} & =\left(\Lambda_{u}\right)^{-1} E_{\overline{y y}}
\end{aligned}
$$

As with $E_{0}$ in the zero-order case, the problem has a unique solution using the matrix $E_{u}$. The solution for the warping and electric potential fields is finally expressed as

$$
\bar{Y}_{1}=\bar{Y}_{1 \chi} \chi+\bar{Y}_{1 \hat{T}} \hat{T}=-E_{u}^{-1}\left(\beta_{\bar{y}} A_{1} z_{0}+\beta_{\bar{y}} b_{1} \hat{T}-\beta_{\bar{y}^{\prime}} A_{1} z_{0}^{\prime}\right)
$$

\section{Second-Order Solution}

The next term in expansion (31), $\bar{Y}_{2}$, is computed from the minimization of the next even term in the expansion of the enthalpy (34), $\mathcal{H}_{4}$, and orthogonality constraints (28) and (30). It is

$$
\mathcal{H}_{4}=\frac{1}{2}\left\{\begin{array}{lll}
z_{0}^{T} & z_{1}^{T} & z_{2}^{T}
\end{array}\right\} \cdot\left[\begin{array}{ccc}
0 & 0 & A_{2} \\
0 & A_{2} & A_{1} \\
A_{2} & A_{1} & A_{0}
\end{array}\right] \cdot\left\{\begin{array}{l}
z_{0} \\
z_{1} \\
z_{2}
\end{array}\right\}+z_{2}^{T} b_{2} \hat{T}
$$

and if $\mu_{2}$ are the Lagrange multipliers that enforce the orthogonality constraints, the Euler equation for the problem is

$$
\begin{gathered}
E_{\overline{y y}} \bar{Y}_{2}+\beta_{\bar{y}}\left(A_{1} z_{1}+A_{2} z_{0}+b_{2} \hat{T}\right)-\beta_{\bar{y}^{\prime}}\left(A_{1} z_{1}^{\prime}+A_{2} z_{0}^{\prime}+b_{2} \hat{T}^{\prime}\right) \\
=H_{\overline{y y}} \Psi_{\epsilon}^{\bar{y}} \mu_{2}^{\epsilon}+H_{\overline{y y}} \Psi_{q}^{\bar{y}} \mu_{2}^{q}
\end{gathered}
$$

As in the first-order solution, the Lagrange multipliers are computed by premultiplying this expression with the transposes of $\Psi_{\epsilon}^{y}$ and $\Psi_{q}^{\bar{y}}+\bar{Y}_{0 q}$, respectively, followed by the orthogonality conditions. The final solution has the form

$$
\begin{aligned}
\bar{Y}_{2}= & \bar{Y}_{2 \hat{\epsilon}} \hat{\epsilon}+\bar{Y}_{2 \hat{\epsilon}^{\prime}} \hat{\epsilon}^{\prime}+\bar{Y}_{2 q} q+\bar{Y}_{2 q^{\prime}} q^{\prime}+\bar{Y}_{2 q^{\prime \prime}} q^{\prime \prime}+\bar{Y}_{2 v} v \\
& +\bar{Y}_{2 v^{\prime}} v^{\prime}+\bar{Y}_{2 v^{\prime \prime}} v^{\prime \prime}+\bar{Y}_{2 T} \hat{T}+\bar{Y}_{2 T^{\prime}} \hat{T}^{\prime}
\end{aligned}
$$

\section{$n$ th-Order Solution $(n>2)$}

A recursive solution scheme of the asymptotic expansion of $\bar{Y}$ in Eq. (31) can be set up. Assume that the expansion is known up to order $n-1$, then the next term $\bar{Y}_{n}$ will be obtained from minimizing $\mathcal{H}_{2 n}$ of enthalpy (34), with orthogonality constraints (28) and (30). Its nonzero terms are

$$
\mathcal{H}_{2 n}=\frac{1}{2}\left\{z_{n-2}^{T} \quad z_{n-1}^{T} \quad z_{n}^{T}\right\} \cdot\left[\begin{array}{ccc}
0 & 0 & A_{2} \\
0 & A_{2} & A_{1} \\
A_{2} & A_{1} & A_{0}
\end{array}\right] \cdot\left\{\begin{array}{c}
z_{n-2} \\
z_{n-1} \\
z_{n}
\end{array}\right\}
$$

Note that the temperature, which is a prescribed field in the cross section, does not explicitly appear in Eq. (53). Taking $\mu_{n}$ as the Lagrange multipliers that enforce the constraints, the Euler equation in $\bar{Y}_{n}$ is

$$
\begin{aligned}
& E_{\overline{y y}} \bar{Y}_{n}+\beta_{\bar{y}}\left(A_{2} z_{n-2}+A_{1} z_{n-1}\right)-\beta_{\bar{y}^{\prime}}\left(A_{2} z_{n-2}^{\prime}+A_{1} z_{n-1}^{\prime}\right) \\
& =H_{\overline{y y}} \Psi_{\epsilon}^{\bar{y}} \mu_{n}^{\epsilon}+H_{\overline{y y}} \Psi_{q}^{\bar{y}} \mu_{n}^{q}
\end{aligned}
$$

When the Lagrange multipliers are solved as shown, the general solution to the expansion of the cross-sectional unknown warping and electric potential, for $n>2$, is given by

$$
\begin{aligned}
\bar{Y}_{n} \equiv & \sum_{k=0}^{n-1}\left(\bar{Y}_{\chi_{\chi^{(k)}}} \chi^{(k)}+\bar{Y}_{T_{T^{(k)}}} \hat{T}^{(k)}\right)=-E_{u}^{-1}\left[\beta_{\bar{y}}\left(A_{2} z_{n-2}+A_{1} z_{n-1}\right)\right. \\
& \left.-\beta_{\bar{y}^{\prime}}\left(A_{2} z_{n-2}^{\prime}+A_{1} z_{n-1}^{\prime}\right)\right]
\end{aligned}
$$

where $(k)$ is the $k$ th derivative along the longitudinal direction of the given variable.

\section{Electroelastic Stiffness Matrix and Hygrothermal Forces}

The warping and electric potential have been evaluated as function of the one-dimensional variables in Eqs. (38), (49), and (52). When those expressions are substituted in the cross-sectional electric enthalpy (25), it can be now written as a quadratic functional in the long-scale electroelastic variables with a forcing term due to the distribution of temperatures. The components of the asymptotic expansion of the cross-sectional electric enthalpy are then

$$
\begin{aligned}
2 \mathcal{H}_{0} & =z_{0}^{T} A_{0} z_{0} \\
2 \mathcal{H}_{1} & =z_{0}^{T} A_{1} z_{0}+2 z_{0}^{T} b_{1} \hat{T} \\
2 \mathcal{H}_{2} & =z_{0}^{T} A_{2} z_{0}+2 z_{0}^{T} b_{2} \hat{T}+\left(2 z_{1}^{T}-\bar{Y}_{1}^{T} \beta_{\bar{y}}\right)\left(A_{1} z_{0}+b_{1} \hat{T}\right) \\
& +\bar{Y}_{1}^{T} \beta_{\bar{y}^{\prime}} A_{1} z_{0}^{\prime} \\
2 \mathcal{H}_{3} & =2 z_{1}^{T}\left(A_{2} z_{0}+b_{2} \hat{T}\right)+z_{1}^{T} A_{1} z_{1}+2\left(z_{2}^{T}-\bar{Y}_{2}^{T} \beta_{\bar{y}}\right)\left(A_{1} z_{0}+b_{1} \hat{T}\right) \\
& +2 \bar{Y}_{2}^{T} \beta_{\bar{y}}^{\prime} A_{1} z_{0}^{\prime} \\
2 \mathcal{H}_{4} & =z_{1}^{T} A_{2} z_{1}+\left(2 z_{2}^{T}-\bar{Y}_{2}^{T} \beta_{\bar{y}}\right)\left(A_{2} z_{0}+A_{1} z_{1}+b_{2} \hat{T}\right) \\
& +\bar{Y}_{2}^{T} \beta_{\bar{y}^{\prime}}\left(A_{1} z_{1}^{\prime}+A_{2} z_{0}^{\prime}+b_{2} \hat{T}^{\prime}\right)
\end{aligned}
$$

and, for $n>2$,

$$
\begin{aligned}
2 \mathcal{H}_{2 n-1}= & 2 z_{n-1}^{T} A_{2} z_{n-2}+z_{n-1}^{T} A_{1} z_{n-1}+2 z_{n}^{T} A_{1} z_{n-2} \\
& -2 \bar{Y}_{n}^{T}\left[\beta_{\bar{y}}\left(A_{1} z_{n-2}-A_{2} z_{n-3}\right)-\beta_{\bar{y}^{\prime}}\left(A_{1} z_{n-2}^{\prime}+A_{2} z_{n-3}^{\prime}\right)\right] \\
2 \mathcal{H}_{2 n} & =z_{n-1}^{T} A_{2} z_{n-1}+2 z_{n}^{T}\left(A_{2} z_{n-2}+A_{1} z_{n-1}\right) \\
& -\bar{Y}_{n}^{T}\left[\beta_{\bar{y}}\left(A_{1} z_{n-1}+A_{2} z_{n-2}\right)-\beta_{\bar{y}^{\prime}}\left(A_{1} z_{n-1}^{\prime}+A_{2} z_{n-2}^{\prime}\right)\right]
\end{aligned}
$$

After summation of the terms of the expansion, one gets

$$
\begin{aligned}
\mathcal{H}= & \left(\begin{array}{lll}
\chi^{T} & \cdots & \chi^{(N)}
\end{array}\right) \cdot\left[\frac{1}{2} S^{N} \cdot\left(\begin{array}{c}
\chi \\
\vdots \\
\chi^{(N)}
\end{array}\right)+F^{N} \cdot\left(\begin{array}{c}
\hat{T} \\
\vdots \\
\hat{T}^{(N)}
\end{array}\right)\right] \\
& +\mathcal{O}\left(\hat{h}^{2 N+1}\right)
\end{aligned}
$$

where $S$ is the $n$ th-order electroelastic stiffness matrix and $F$ is the $n$ th-order hygrothermal force.

Equation (58) gives the general form for the asymptotic approximation up to an arbitrary order of the enthalpy per unit length. For practical purposes, a second-order expansion describes well the 
cross-sectional characteristics of beams with initial twist and curvature. The second-order approximation to the cross-sectional electric enthalpy is

$$
\begin{gathered}
\mathcal{H}=\mathcal{H}^{4}\left(\chi, \chi^{\prime}, \chi^{\prime \prime} ; \hat{T}, \hat{T}^{\prime}, \hat{T}^{\prime \prime}\right)+\mathcal{O}\left(\hat{h}^{5}\right)=\mathcal{H}^{4}\left(\hat{\epsilon}, \hat{\epsilon}^{\prime}, \hat{\epsilon}^{\prime \prime}, q, q^{\prime}, q^{\prime \prime}\right. \\
\left.q^{\prime \prime \prime}, v, v^{\prime}, v^{\prime \prime}, v^{\prime \prime \prime} ; \hat{T}, \hat{T}^{\prime}, \hat{T}^{\prime \prime}\right)+\mathcal{O}\left(\hat{h}^{5}\right)
\end{gathered}
$$

The last expression defines 105 different coefficient matrices. They correspond to the most general second-order asymptotic reduction of an active structure with finite section deformation modes and thermal effects. As particular applications, two typical cases are considered next. The notation $z_{n p}=\partial z_{n} / \partial p$ is used, with $z_{n}$ being the coefficients of expansion (32). First, consider the second-order classical description of a passive structure with constant thermal excitation. The variables in this case are $\left(\hat{\epsilon}, \hat{\epsilon}^{\prime}, \hat{\epsilon}^{\prime \prime} ; T\right)$, which corresponds to a refined version of classical beam theory. The resulting model would account for end effects or could used to compute the transverse shear stiffness. ${ }^{26}$ The stiffness constants in this case are given by

$$
\begin{aligned}
S_{\hat{\epsilon} \hat{\epsilon}}^{4}= & \hat{h}^{2}\left(z_{0 \hat{\epsilon}}^{T} A_{2} z_{0 \hat{\epsilon}}+z_{1 \hat{\epsilon}}^{T} A_{1} z_{0 \hat{\epsilon}}\right)+\hat{h}^{3}\left[z_{0 \hat{\epsilon}}^{T} A_{2} z_{1 \hat{\epsilon}}+z_{1 \hat{\epsilon}}^{T}\left(A_{2} z_{0 \hat{\epsilon}}\right.\right. \\
& \left.\left.+A_{1} z_{1 \hat{\epsilon}}\right)\right]+\hat{h}^{4}\left[z_{1 \hat{\epsilon}}^{T} A_{2} z_{1 \hat{\epsilon}}+z_{2 \hat{\epsilon}^{\prime}}^{T}\left(A_{2} z_{0 \hat{\epsilon}}+A_{1} z_{1 \hat{\epsilon}}\right)\right] \\
S_{\hat{\epsilon} \hat{\epsilon}^{\prime}}^{4}= & \hat{h}^{3}\left(z_{0 \hat{\epsilon}}^{T} A_{2} z_{1 \hat{\epsilon}^{\prime}}+z_{1 \hat{\epsilon}}^{T} A_{1} z_{1 \hat{\epsilon}^{\prime}}\right)+\hat{h}^{4}\left[z_{0 \hat{\epsilon}}^{T} A_{2} z_{2 \hat{\epsilon}^{\prime}}+z_{1 \hat{\epsilon}}^{T}\left(A_{2} z_{1 \hat{\epsilon}^{\prime}}\right.\right. \\
& \left.\left.+A_{1} z_{2 \hat{\epsilon}^{\prime}}\right)+\bar{Y}_{2 \hat{\epsilon}}^{T} \beta_{\bar{y}^{\prime}}\left(A_{2} z_{0 \hat{\epsilon}}+A_{1} z_{1 \hat{\epsilon}}\right)\right] \\
S_{\hat{\epsilon} \hat{\epsilon}^{\prime \prime}}^{4} & =\hat{h}^{4}\left(z_{0 \hat{\epsilon}}^{T} A_{2} z_{2 \hat{\epsilon}^{\prime \prime}}+z_{1 \hat{\epsilon}}^{T} A_{1} z_{2 \hat{\epsilon}^{\prime \prime}}\right) \\
S_{\hat{\epsilon}^{\prime} \hat{\epsilon}^{\prime}}^{4} & =\hat{h}^{4}\left[z_{1 \hat{\epsilon}^{\prime}}^{T}\left(A_{2} z_{1 \hat{\epsilon}^{\prime}}+A_{1} z_{2 \hat{\epsilon}^{\prime}}\right)+\bar{Y}_{2 \hat{\epsilon}^{\prime}}^{T} \beta_{\bar{y}^{\prime}}\left(A_{2} z_{0 \hat{\epsilon}}+A_{1} z_{1 \hat{\epsilon}}\right)\right]
\end{aligned}
$$

and the associated hygrothermal forces are

$$
\begin{aligned}
F_{\hat{\epsilon} \hat{T}}^{4} & =\hat{h}^{2}\left(z_{0 \hat{\epsilon}}^{T} b_{2}+z_{1 \hat{\epsilon}}^{T} b_{1}\right)+\hat{h}^{3}\left[z_{0 \hat{\epsilon}}^{T} A_{2} z_{1 \hat{T}}+z_{1 \hat{\epsilon}}^{T}\left(A_{1} z_{1 \hat{T}}+b_{2}\right)\right] \\
& +\hat{h}^{4}\left[z_{1 \hat{\epsilon}}^{T} A_{2} z_{1 \hat{T}}+z_{2 \hat{\epsilon}}^{T}\left(A_{1} z_{1 T}+b_{2}\right)\right] \\
F_{\hat{\epsilon}^{\prime} \hat{T}}^{4} & =\hat{h}^{3}\left[z_{1 \hat{\epsilon}^{\prime}}^{T}\left(b_{2}+A_{1} z_{1 \hat{T}}\right)\right]+\hat{h}^{4}\left[z_{1 \hat{\epsilon}^{\prime}}^{T}\left(A_{2} z_{1 \hat{T}}+A_{1} z_{2 \hat{T}}\right)\right. \\
& \left.+\bar{Y}_{2 \hat{\epsilon}}^{T} \beta_{\bar{y}^{\prime}}\left(b_{2}+A_{1} z_{1 \hat{T}}\right)\right] \\
F_{\hat{\epsilon}^{\prime \prime} \hat{T}}^{4} & =\hat{h}^{4}\left[z_{2 \hat{\epsilon}^{\prime \prime}}^{T}\left(b_{2}+A_{1} z_{1 \hat{T}}\right)\right]
\end{aligned}
$$

As a second case, consider a first-order coupled solution without thermal forces and finite section modes. This solution models coupled electroelastic properties per unit length for rapidly varying longitudinal fields. The variables in this case are $\left(\hat{\epsilon}, \hat{\epsilon}^{\prime}, v, v^{\prime}, v^{\prime \prime}\right)$, and the nonzero stiffness submatrices are

$$
\begin{aligned}
& S_{\hat{\epsilon} \hat{\epsilon}}^{2}=\hat{h}^{2}\left(z_{0 \hat{\epsilon}}^{T} A_{2} z_{0 \hat{\epsilon}}+z_{1 \hat{\epsilon}}^{T} A_{1} z_{0 \hat{\epsilon}}\right) \\
& S_{v v}^{2}=\hat{h}^{0}\left(z_{0 v}^{T} A_{0} z_{0 v}\right)+\hat{h}^{1}\left(z_{0 v}^{T} A_{1} z_{0 v}\right)+\hat{h}^{2}\left(z_{0 v}^{T} A_{2} z_{0 v}+z_{1 v}^{T} A_{1} z_{0 v}\right) \\
& S_{\hat{\epsilon} v}^{2}=\hat{h}^{1}\left(z_{0 \hat{\epsilon}}^{T} A_{1} z_{0 v}\right)+\hat{h}^{2}\left(z_{0 \hat{\epsilon}}^{T} A_{2} z_{0 v}+z_{1 \hat{\epsilon}}^{T} A_{1} z_{0 v}\right) \\
& S_{\hat{\epsilon} v^{\prime}}^{2}=\hat{h}^{2}\left[z_{0 \hat{\epsilon}}^{T}\left(A_{2} z_{0 v^{\prime}}+A_{1} z_{1 v^{\prime}}\right)+\bar{Y}_{1 \hat{\epsilon}}^{T} \beta_{\bar{y}^{\prime}} A_{1} z_{0 v}\right] \\
& S_{\hat{\epsilon}^{\prime} v}^{2}=\hat{h}^{2}\left(z_{1 \hat{\epsilon}^{\prime}}^{T} A_{1} z_{0 v}\right) \\
& S_{v v^{\prime}}^{2}=\hat{h}^{1}\left(z_{0 v}^{T} A_{1} z_{0 v^{\prime}}\right)+\hat{h}^{2}\left[z_{0 v}^{T}\left(A_{2} z_{0 v^{\prime}}+A_{1} z_{1 v^{\prime}}\right)+\bar{Y}_{1 v}^{T} \beta_{\bar{y}^{\prime}} A_{1} z_{0 v}\right] \\
& S_{v^{\prime} v^{\prime}}^{2}=\hat{h}^{2}\left\{z_{0 v^{\prime}}^{T} A_{2} z_{0 v^{\prime}}+\left[\bar{Y}_{1 v^{\prime}}^{T}\left(\beta_{\bar{y}} A_{1} z_{0 v^{\prime}}+\beta_{\bar{y}^{\prime}} A_{1} z_{0 v}\right)\right]_{s}\right\} \\
& S_{v v^{\prime \prime}}^{2}=\hat{h}^{2}\left(z_{0 v}^{T} A_{1} z_{1 v^{\prime \prime}}\right)
\end{aligned}
$$

\section{Recovery of Three-Dimensional Stress, Strain, Displacement, and Electric Fields}

The methodology for the asymptotic reduction of the threedimensional electrothermoelastic problem to a set of stiffness coefficients along the reference line has been already shown. During the process, the warping and induced electric potential crosssectional fields corresponding to unit values of each component of the one-dimensional state variable $\chi$ and its longitudinal derivatives were also computed. These fields can be identified as the $n$ th-order $(\mathrm{WIC})_{n}$ and (EIC $)_{n}$, respectively:

$$
\mathrm{WIC}_{n}=\frac{\partial \bar{W}}{\partial \chi^{(n)}}, \quad \mathrm{EIC}_{n}=\frac{\partial \bar{\Phi}}{\partial \chi^{(n)}}
$$

When these coefficients are used, the process of recovery of the displacement and electric fields on the deformed three-dimensional domain, $\Omega$, is a straightforward one (Fig. 1). The stiffness coefficients and thermal forces of equation (58) (where crosssectional inertia properties are also needed for a dynamic response) reduce the system to the reference line. The subsequent $n$ th-order one-dimensional problem is then solved in the variables $\left(\chi, \chi^{\prime}, \ldots, \chi^{(n)}\right)$, for a given the external excitation and initial conditions. Let $\chi(t), \chi^{\prime}(t), \ldots, \chi^{(n)}(t)$ be the one-dimensional state at time $t$. The three-dimensional fields at a given cross section are then evaluated as the superposition of the one-dimensional response (rigid-body displacement of the original cross section, assumed deformation modes, and the assumed potential at electrodes), plus the contribution due to the influence coefficients. The column matrix of the recovered displacement field is

$$
\begin{aligned}
X= & R\left(x_{1}\right)+\xi+\sum_{m} \psi_{q_{m}}\left(x_{2}, x_{3}\right) q_{m}\left(x_{1}\right) \\
& +\sum_{n} \mathrm{WIC}_{n}\left(x_{2}, x_{3}\right) \chi^{(n)}\left(x_{1}\right)+\mathcal{O}\left(\hat{h}^{N+1}\right)
\end{aligned}
$$

where vectors have been expressed in its components in the deformed frame $B$ and

$$
\xi=\left\{\begin{array}{lll}
0 & x_{2} & x_{3}
\end{array}\right\}^{T}
$$

and the recovered distribution of electric potential is

$$
\begin{aligned}
\phi(x) & =\sum_{m} \psi_{v_{m}}\left(x_{2}, x_{3}\right) \cdot v_{m}\left(x_{1}\right) \\
& +\sum_{n} \operatorname{EIC}_{n}\left(x_{2}, x_{3}\right) \cdot \chi^{(n)}\left(x_{1}\right)+\mathcal{O}\left(\hat{h}^{N+1}\right)
\end{aligned}
$$

These expressions provide an asymptotic description of the state after deformation in the three-dimensional domain.

\section{Sample Cases}

The former theoretical derivations have been implemented into the computer code UM/VABS. Two simple cases are now considered to illustrate typical applications in which UM/VABS is the only alternative to detailed three-dimensional finite element analysis: an active beam with orthotropic properties and significant shear effects and the analysis of nonclassical deformations an active box-beam to distributed prescribed actuation.

\section{Active Orthotropic Beam with Square Cross Section}

This numerical test case studies an active prismatic beam with square cross section of length $a$ and two-dimensional orthotropic material properties. Several key points are of interest in the analysis of this configuration: 1) This type of solid construction is known to have significant contribution of shear in the strain energy, which needs to be accounted for appropriately. 2) At the same time, orthotropic properties generate significant elastic couplings (in this case, twist-bending and extension-shear), and elasticity solutions are no longer readily available as in the case of isotropic beams. 3) Finally, analysis of electroelastic couplings under elastic and electric excitations is particularly illustrative for this simple model, and it is included in the discussion. All of these effects are investigated using the present theory and are compared with MSC.NASTRAN 
Fig. 4 Layout of active prismatic orthotropic beam.
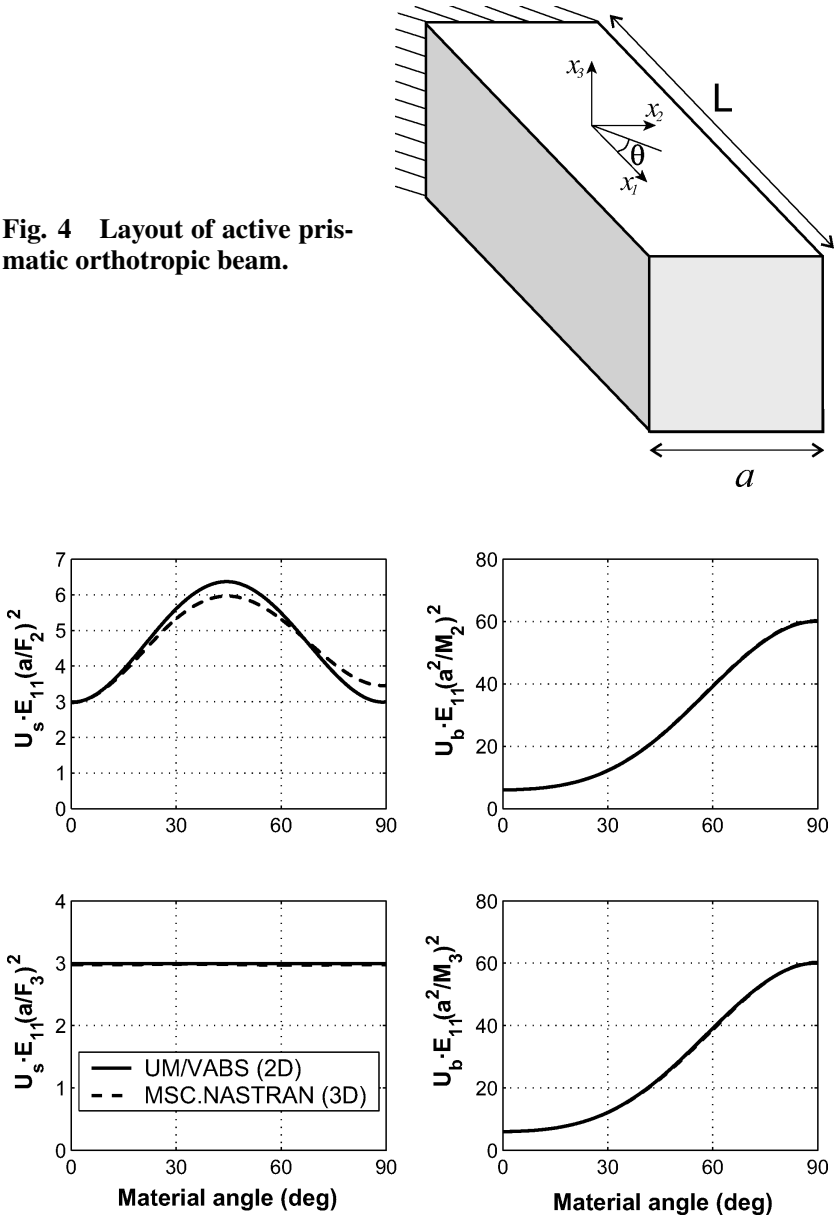

Fig. 5 Nondimensional strain energy per unit length due to shear forces and bending moments.

static elastic results on three-dimensional finite element models. The definition of this test case is shown in Fig. 4.

Properties correspond to a homogeneous orthotropic material at an angle $\theta$ in the $x_{1}-x_{2}$ plane, with elastic constants $E_{22}=$ $E_{33}=E_{11} / 10, \quad G_{12}=G_{13}=G_{23}=E_{11} / 5, \quad v_{12}=v_{13}=0.25$, and $v_{23}=0.3$. Material properties also include a significant orthotropic piezoelectric effect, with nonzero permittivity constants $\varepsilon_{11}=\varepsilon_{22}=\varepsilon_{33} / 10$, and inverse piezoelectric constants defined as $d_{3,33}=\delta_{c} \sqrt{ }\left(\varepsilon_{33} / E_{11}\right), d_{3,11}=-d_{3,33} / 2, d_{3,22}=-d_{3,33} / 2$, and $d_{1,13}=d_{2,23}=d_{3,33} / 4$. Here $\delta_{c}$ is a nondimensional coupling parameter between the elastic and electric field. For piezoelectric materials, this coupling is usually weak, and $\delta_{c}$ is a small number. Transverse shear effects can be important in the elastic response of these constructions and are included in the analysis. For that purpose, an approximate solution can be found within the context of the present formulation if the shear deformation is geometrically approximated with the nondimensional finite section deformation modes

$$
\psi_{q_{\alpha}}=\left\{\begin{array}{lll}
\hat{x}_{\alpha} & 0 & 0
\end{array}\right\}^{T}
$$

The corresponding amplitudes are $q_{\alpha}=2 \gamma_{1 \alpha}$, that is, the transverse shear strains, as can be seen from Eqs. (6) and (10).

Theory of elasticity only provides a closed-form solution for transverse shear effects in isotropic materials. ${ }^{30,31}$ To define a reference for comparison, three-dimensional finite element models have been created in MSC.NASTRAN on this configuration. A beam of length $L=15 a$ is modeled as a three-dimensional solid, being clamped on one end and with applied constant distributed loads on the free end. After a linear static analysis, integration of the strain distribution in the cross section at $x_{1}=L / 2$ yields the energy per unit length for the Saint-Venant interior solution of the beam. Figure 5 shows the strain energy per unit length due to internal shear forces in both directions (shear energy $U_{s}$ ), and due to internal bending moments (bending energy $U_{b}$ ), as computed from 1) the integration of three-dimensional strains in the finite element model in MSC.NASTRAN and 2) the elastic stiffness coefficients using in UM/VABS with the approximation of Eq. (67). In both cases, the cross section was discretized using $25 \times 25$ elements. A very good agreement is found between both solutions. A more detailed comparison between the asymptotic analysis using finite section modes and the three-dimensional FEM solution can be performed by looking at the cross-sectional distribution of shear strain. Figure 6 shows this comparison for given shear forces $F_{\alpha}$ between the interior solution in the static response to tip shear forces in an MSC.NASTRAN model using three-dimensional hexahedral elements and the cross-sectional solution using UM/VABS with finite section mode for shear. Results were nondimensionalized using the following reference measure for shear strain:

$$
\Gamma_{1 \beta}^{*}\left(F_{\alpha}\right)=F_{\alpha} / 2 G_{1 \beta} a^{2}
$$

As can be observed from the comparison, UM/VABS can accurately evaluate the stress distribution in the interior solution of the anisotropic beam. Note that, because a two-dimensional problem is solved, the UM/VABS solution is computationally much more efficient that the finite-element solution of the complete threedimensional problem.

Finally, if the piezoelectric characteristics of the cross section are also considered, it is possible to solve the coupled electroelastic problem in the cross section either for elastic or electric loads. As an example, Fig. 7 shows the open-circuit cross-sectional influence coefficients for the electric potential with respect to unit axial strain, $\gamma_{11}=1$, and unit nondimensional twist and bending curvatures, $a \kappa_{i}=1$. The coupling parameter is $\delta_{c}=10^{-6}$, and results are given for two material orientations, $\theta=0$ and $\theta=30 \mathrm{deg}$, and with respect to a reference potential defined as $\phi_{\text {ref }}=\left(\varepsilon_{33}\right)^{-1} a E_{11} d_{3,11}$. In all cases, $\phi=0$ was set at $\left(2 x_{2} / a, 2 x_{3} / a\right)=(-1,-1)$. The values for $\theta=0$ deg in Fig. 7 can be easily estimated from the piezoelectric constitutive relations, assuming that the induced potential does not modify the strain distributions (negligible electroelastic coupling) and the potential is a first integral of the electric field. For $\theta=30 \mathrm{deg}$, that solution is modified by the effect of the material anisotropy.

As the electroelastic coupling parameter $\delta_{c}$ increases, part of the work applied to deform the structure is stored as electric energy and the values of the elastic stiffness constants move up. Figure 8 shows this stiffening effect for two material angles, $\theta=0$ and $30 \mathrm{deg} . S_{i j}^{0}$ are the stiffness constants without coupling, $\delta_{c}=0$, and $\Delta S_{i j}=S_{i j}-S_{i j}^{0}$. Note that the stiffening effect becomes only appreciable for $\delta_{c}>0.1$, which is much higher than typical values of the constant in piezoelectric materials. Note also that the torsion constant is not affected with $\theta=0 \mathrm{deg}$, in agreement with results in Fig. 7. As one could expect in a homogeneous fully actuated case, changes in the coupling parameter $\delta_{c}$ do not generate a modification of the shape of the electric potential distribution for a given elastic deformation. Their effect is reduced to a constant factor $f$ in their values, which can be defined as $\phi\left(\delta_{c}\right) / \phi_{\text {ref }}\left(\delta_{c}\right)=f\left(\delta_{c}\right) \phi(0) / \phi_{\text {ref }}(0)$. Figure 9 shows the variation of this factor for each elastic deformation and for two material angles. As before, for $\delta_{c}<0.1$ the coupling is negligible, $f=1$.

Consider finally a rectangular cross section (width $a$ and height/thickness $b$ ) subject to an electric field induced by two electrodes of length $l$ and with voltage $\pm V$, applied as shown in Fig. 10 . In the current formulation, electrode configurations are defined by changing the applied potential $\Psi_{v}^{\phi}$ in Eq. (24), with its corresponding amplitude being the amplitude of the applied voltage $V$.

For small piezoelectric coupling, the induced electric field by an applied excitation can be determined in an approximate manner by solving the equations of electrostatics. Consider first the crosssectional problem with homogeneous material properties as defined earlier and with electrodes covering the upper and lower surfaces, 

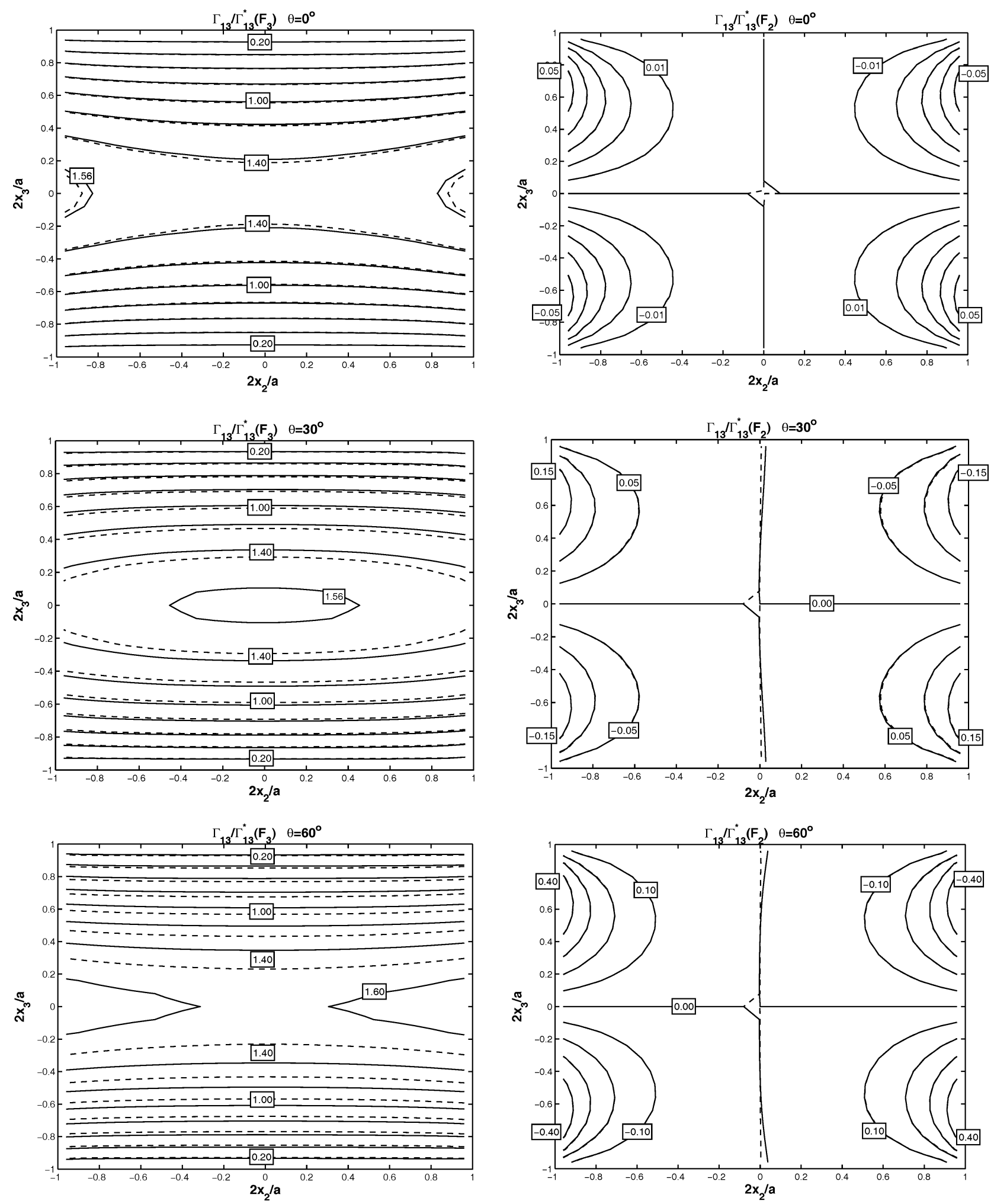

Fig. 6 Distribution of shear strains in orthotropic square cross section for different material orientation angles $\theta$ and component of shear force $F_{\alpha}$ : , UM/VABS and - - -, MSC.NASTRAN.

$l=a$. The boundary conditions to the two-dimensional electrostatic problem are then

$$
\begin{array}{ccc}
\phi= \pm V & \text { at } & x_{2}= \pm b / 2 \\
D_{2}=0 & \text { at } & x_{3}= \pm a / 2
\end{array}
$$

and the solution is given by a constant electric field $E_{3}=\Delta V / b$. Therefore, for homogeneous material properties, negligible elec- troelastic coupling parameter, and surfaces fully covered by electrodes, the usual assumption in the prescribed field solution (thermal analogy) is the exact solution to the problem. With this, the capacitance per unit length $C$ can be obtained from the total electric charge in the cross section under the applied voltage

$$
C=\frac{\int_{-b / 2}^{b / 2} D_{3} \mathrm{~d} x_{3}}{\Delta V}=\varepsilon_{33} \frac{l}{b}
$$



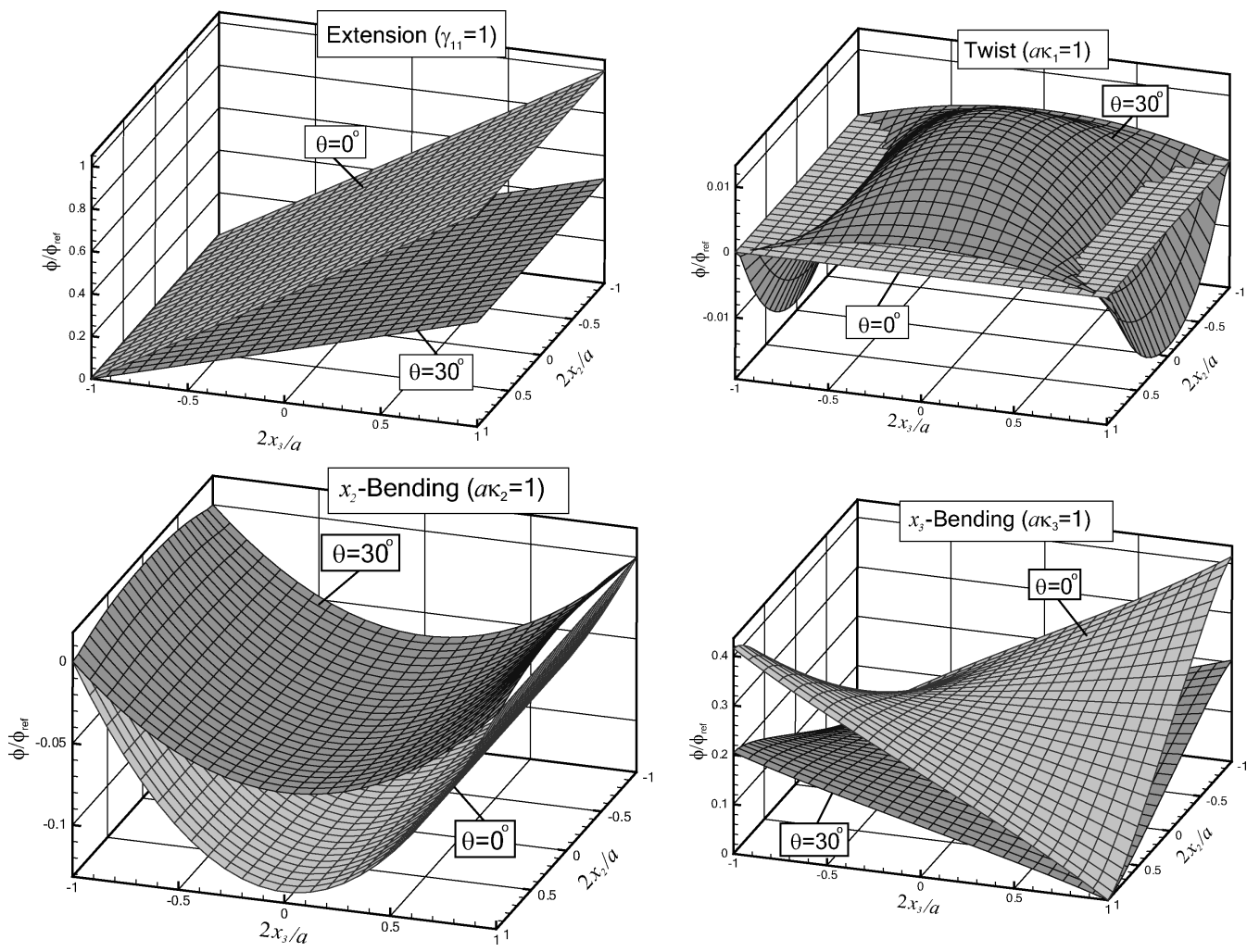

Fig. 7 Electric potential distribution in the square orthotropic cross section corresponding to unit deformations for weak coupling, $\delta_{c}=10^{-6}$ and two material angles, $\theta=0$ and $30 \mathrm{deg}$.
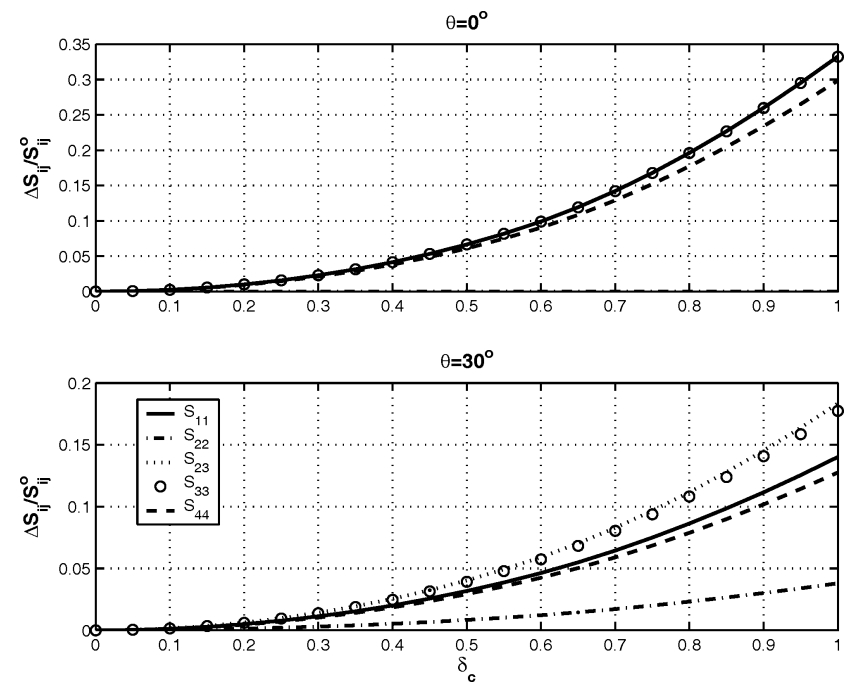

Fig. 8 Stiffening effect of piezoelectric coupling for two material angles.

and this serves to define the $S_{v v}$ component in the electroelastic stiffness matrix. If the amplitude of the electric excitation modes is defined as $V$, the contribution of the electric field to the crosssectional enthalpy is defined with the following coefficient matrix:

$$
S_{v v}^{0}=-4 C=-4 \varepsilon_{33}(l / b)
$$

This value could be equally obtained by substituting the constant electric field into the electric contribution to the cross-sectional enthalpy (16). Note that, for the current definition of the material properties, $S_{v v}$ is independent of the material orientation $\theta$. The total force per unit length induced by this electric field can easily be obtained for $\theta=0 \mathrm{deg}$. Then, a constant axial strain appears with value
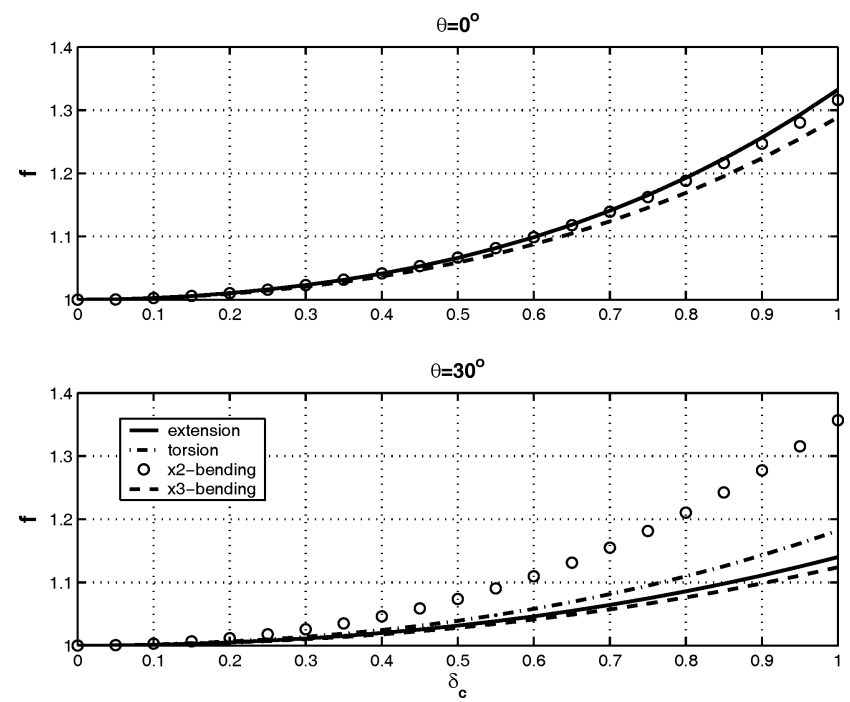

Fig. 9 Effect of piezoelectric coupling in magnitude of induced electric potential field.

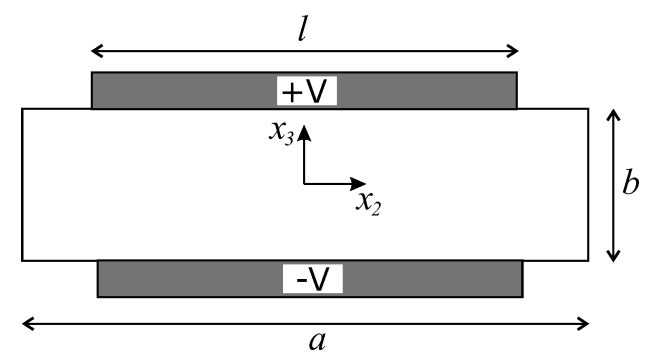

Fig. 10 Geometrical parameters in rectangular cross section with electrodes. 
$\Gamma_{11}(0 \mathrm{deg})=d_{3,11} E_{3}$, whose integration gives a net axial active force of value

$$
F_{1}^{(\alpha)}(0 \mathrm{deg})=E_{11} l d_{3,11} \Delta V
$$

This force defines the corresponding coupling term in the $S_{\epsilon v}$ submatrix of the electroelastic stiffness as

$$
S_{1 v}^{0}(0 \mathrm{deg})=2 E_{11} l d_{3,11}
$$

The situation described by Eqs. (71-73) is based on the small value of $\delta_{c}$, which decouples the elastic and electric solutions, and on the simplification of electrostatic boundary conditions when $l=a$. For a general case, a numerical solution is needed, and it is provided by the cross-sectional formulation introduced here. As an example, consider the case of electrodes not completely covering the surface, $l<a$. For given section thickness $b$, the ratio $S_{v v} / S_{v v}^{0}$ increases with $a / l$, up to a limit value in which the effect of the side walls is not noticeable. This is shown in Fig. 11 for $b / l=1$.

Figure 12 shows the variation with the section thickness $b$ of $S_{v v}$ for two electrode configurations. In the first of them (open configuration), the part of the upper and bottom surfaces that is not covered by the electrodes, $x_{2}>l$, is left in open circuit. In the second case, $V=0$, short-circuit conditions are enforced on the same zones. In both cases, $a$ is taken large enough to have little influence in the result, $l / a \rightarrow 0$. It was shown before that an increase in the electroelastic coupling parameter $\delta_{c}$ produces elastic stiffening of the structure. Analogously, the electric compliance (capacitance) of the cross section will be affected by this parameter. Figure 13 shows this variation for a large value of the coupling parameter, $\delta_{c}=0.3162$, different elastic material angles for $b / l=1$, and both limits in the $l / a$ ratio. The results are given with respect to the corresponding capacitance in the uncoupled problem, $S_{v v}^{0}$. It is seen above that $S_{v v}^{0}$ is independent of the material angle. However, the results from Fig. 13 indicate that this is no longer the case in the presence of significant coupling. The cross-sectional capacitance decreases when there is interaction between the electric and elastic properties, although the changes in its magnitude are relatively small (less that $2.5 \%$ ), and it can be neglected as a first approximation to the solution of practical problems.

\section{Platelike Motions in Active Box-Beams with Prescribed Electric Field}

This second numerical test case corresponds to an active composite box-beam with length $\times$ width $\times$ height equal to $20 a \times 2 a \times a$. A dominant dimension can be identified in this structure, and due to this a one-dimensional model is desirable for its analysis. The thin-wall construction, however, implies the likely appearance of platelike components in the deformation under loading, which is particularly important when the excitation comes from distributed embedded actuators. This section illustrates how the platelike motions of a thinwalled beam can be easily included in a one-dimensional model using finite section modes. The composite construction at each

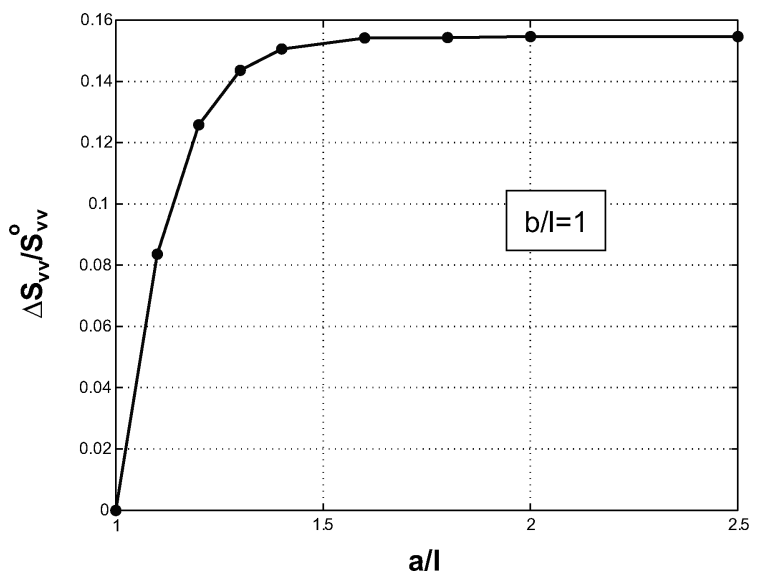

Fig. 11 Cross-sectional capacitance for varying width and fixed thickness-to-electrode length ratio.

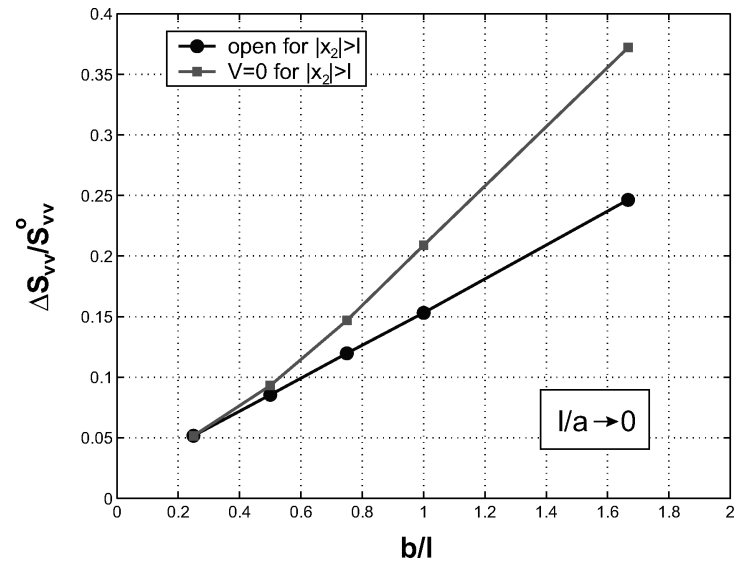

Fig. 12 Change in cross-sectional capacitance with thickness-toelectrode length ratio.

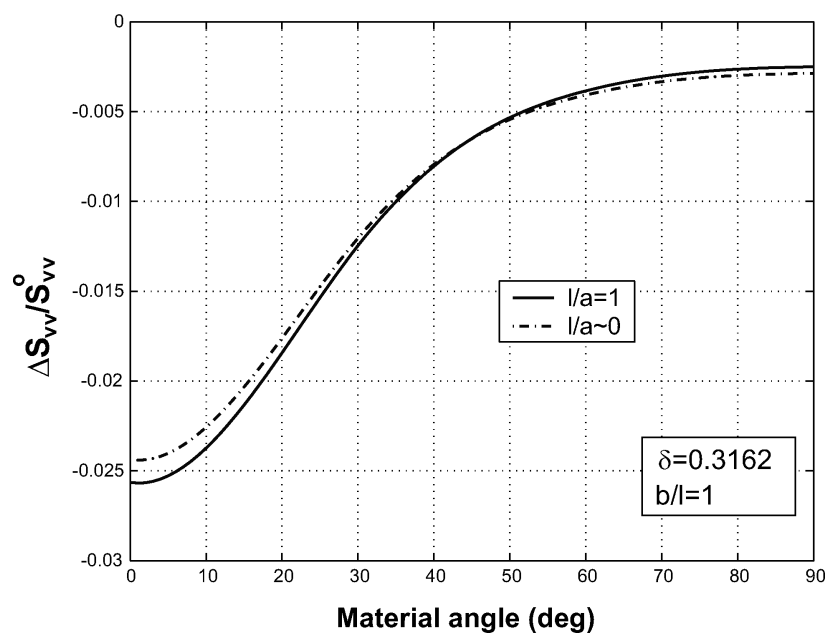

Fig. 13 Variation of cross-sectional capacitance with elastic material angle in coupled case.

wall is made out of four two-dimensional orthotropic plies, each one being $a / 100$ thick, and with elastic constants $E_{22}=E_{11} / 20$, $G_{12}=E_{11} / 10$, and $v_{12}=0.35$. The bond layer has not been included in this model, although there is no restriction in the formulation to account for its effect. (Only a higher-fidelity discretization would be needed. ${ }^{1}$ ) A prescribed through-thickness electric field $E_{3}$ is assumed in this construction, such that the free-strain deformation of a single ply is given by $d_{3,11} E_{3}= \pm \Gamma_{11}^{0}$ in the direction of the fiber (the sign depending on the sign of the electric field) and with $d_{3,22}=-d_{3,11} / 3$ in the transverse direction. The electric field $E_{3}$ is assumed here to be constant within a ply, when corresponds to the usual assumption for the analysis of piezoelectrics in structural applications. ${ }^{3,7,32}$ The prescribed field assumption implies a decoupling between the electric and elastic fields, which in the context of the present theory is equivalent to substitution of the electric field along the polarization axis for a temperature field (the so-called thermal analogy). Four different actuation cases are analyzed here using the thermal analogy for the same laminate construction, as shown in Table 1, where plies are numbered inside out. The positive fiber angle goes along the clockwise direction on each wall.

A finite element model of these configurations was built using shell elements in MSC.NASTRAN. The static response for thermal loads was then computed for each actuation case, and they are shown in Fig. 14. Cases A1 and A2 can be easily identified as onedimensional bending and twist responses, and a classical composite beam model will be then enough to capture the response. However, cases A3 and A4 clearly include platelike motions, which, in a one-dimensional structural model, will appear as higher-order components of the deformation. Their characterization can be done 


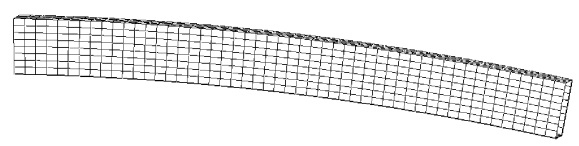
$z L^{Y} x$

Case A1

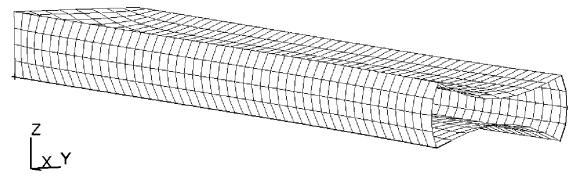

Case A3

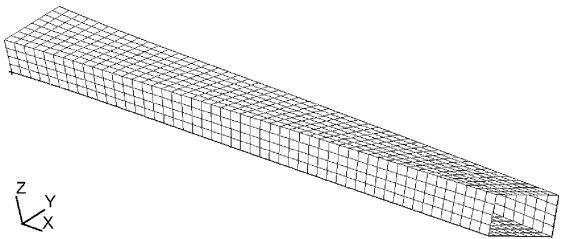

Case A2

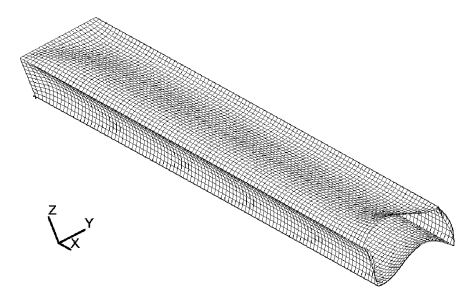

Case A4

Fig. 14 MSC.NASTRAN static response for the box-beam under thermal loads.

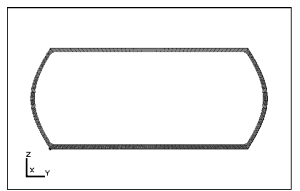

Mode \#11

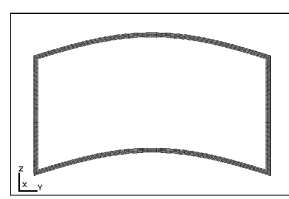

Mode \#14

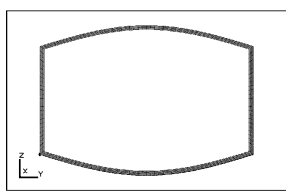

Mode \#12

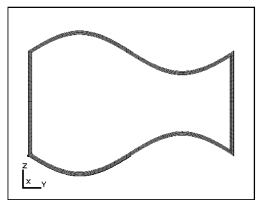

Mode \#22

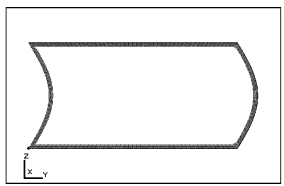

Mode \#13

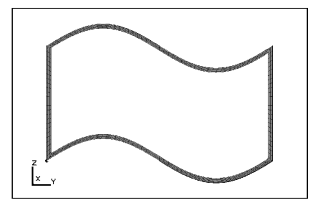

Mode \#24

Fig. 15 UM/VABS finite section modes for platelike motions of active box-beam.

Table 1 Laminate layups and electric actuations: $\Uparrow$, upper wall; $\Downarrow$, bottom; $\Rightarrow$, right; and $\Leftarrow$, left

\begin{tabular}{lcc}
\hline \hline Case & Elastic ply up & Electric actuation \\
\hline A1 & $\left(45 /-45_{2} / 45\right) \Uparrow \Downarrow \Rightarrow \Leftarrow$ & $(+.+.+.+) \Rightarrow ;(-,-,-,-) \Leftarrow$ \\
A2 & $(+.-,-.+) \Rightarrow \Leftarrow$ \\
A3 & & $(-, 0,0,+) \Rightarrow \Leftarrow$ \\
A4 & & $(-/ 0 / 0 /+) \Rightarrow ;(+/ 0 / 0 /-) \Leftarrow$ \\
\hline \hline
\end{tabular}

in the context of the present cross-sectional reduction using finite section modes. For the box-beam under consideration, the reference line is chosen to be along the area centroid of the cross sections, and the deformation modes are defined with harmonic functions in given walls $\left[\psi_{q}=\cos \left(\pi c x_{\alpha} / 2 L_{\alpha}\right)\right.$, with $L_{\alpha}$ the wall length and $\left.c=1,2\right]$, as shown in Fig. 15.

This set of finite section modes satisfies the orthogonality conditions (7). Orthogonality to the rigid-body motions of the cross section $\Psi_{\epsilon}^{w}$ must be finally imposed for them to satisfy condition (5), and this is easily done by subtracting their rigid-body components (motions along $x_{2}$ and $x_{3}$ for modes 13 and 14, respectively, and in-plane rotation for mode 24). The resulting set of modes defines a unique expansion of the cross-sectional warping field.

The subsequent first-order cross-sectional analysis yields a $16 \times 16$ stiffness matrix $S$ associated to the set of one-dimensional variables $\left\{\epsilon, q, q^{\prime}\right\}$, the corresponding generalized thermal forces per unit length $F$, and the influence coefficients of the cross-sectional warping field with respect to the one-dimensional variables. With that information, the one-dimensional reduced model can be built and solved for this configuration as it was done in Ref. 33. Following the general scheme of Fig. 1, the three-dimensional displacement
Table 2 One-dimensional interior solution of active box-beam and maximum error in cross-sectional displacements with respect to MSC.NASTRAN results

\begin{tabular}{lcccc}
\hline \hline Measures & $\mathrm{A} 1$ & $\mathrm{~A} 2$ & $\mathrm{~A} 3$ & $\mathrm{~A} 4$ \\
\hline$a \kappa_{1}^{\infty} / \Gamma_{11}^{0}$ & 0 & -0.966 & 0 & 0 \\
$a \kappa_{3}^{\infty} / \Gamma_{11}^{0}$ & -0.550 & 0 & 0 & 0 \\
$q_{11}^{\infty} /\left(a \Gamma_{11}^{0}\right)$ & 0 & 0 & 6.472 & 0 \\
$q_{12}^{\infty} /\left(2 a \Gamma_{11}^{0}\right)$ & 0 & 0 & -6.173 & 0 \\
$q_{13}^{\infty} /\left(a \Gamma_{11}^{0}\right)$ & 0 & 0 & 0 & 4.015 \\
$q_{22}^{\infty} /\left(2 a \Gamma_{11}^{0}\right)$ & 0 & 0 & 0 & 1.397 \\
$\langle\|\boldsymbol{\Delta} \boldsymbol{d}\|\rangle /\langle\|\boldsymbol{d}\|\rangle, \%$ & 0.0 & 0.0 & 6.9 & 8.2 \\
\hline \hline
\end{tabular}

field is finally constructed from both the cross-sectional and longitudinal results. In this case, because the thermal force $F$ is constant in the axial direction, the Saint-Venant interior solution $\left\{\epsilon_{\infty}, q_{\infty}, q_{\infty}^{\prime}\right\}$ is directly available from the two-dimensional problem as $S^{-1} F$. For this problem, $q_{\infty}^{\prime}=0$, and the nonzero values for each actuation mode are given in Table 2. The last row in Table 2 includes the average value in the cross section of the error $\Delta \boldsymbol{d}$ between the displacement field in the interior solution of the three-dimensional finite element model, $\boldsymbol{d}$, and the displacements after the recovery process in the cross-sectional analysis. The actual cross-sectional warping field in the interior solution can be explicitly computed as $W=W_{\epsilon} \epsilon_{\infty}+W_{q} q_{\infty}$, where $W_{\epsilon}$ and $W_{q}$ are the WIC matrices. Results for cases A3 and A4 are presented in Fig. 16. Figure 16 shows a comparison of the interior solution in the MSC.NASTRAN plate FEM models and the midwall displacements in the cross-sectional 


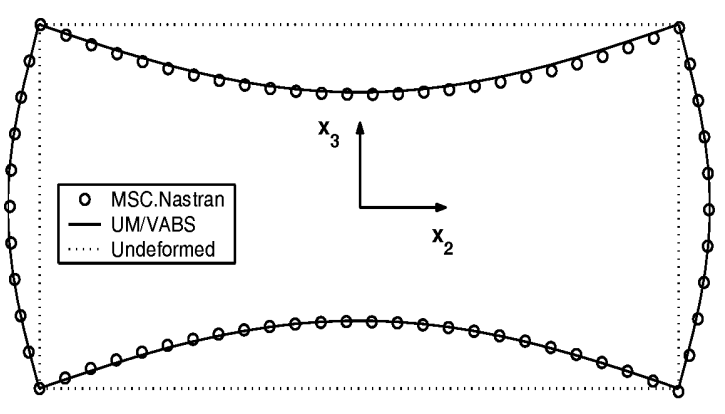

Case A3

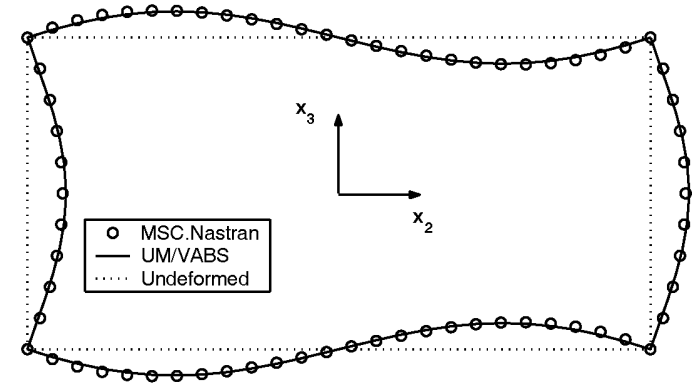

Case A4

Fig. 16 Interior solution for active box-beams A3 and A4 as computed by MSC.NASTRAN plate FEM solution and cross-sectional reduction of UM/VABS using finite section modes.

analysis with finite section modes. The comparison is excellent and shows the effectiveness of the present approach to capture nonclassical deformation modes in slender composite structures.

\section{Conclusions}

This paper presented a comprehensive formulation for the cross-sectional characterization of slender nonhomogeneous and anisotropic structures with embedded sensors and actuators. For this purpose, the coupled equations of electrothermoelasticity in the cross section were solved through an asymptotic approximation. The numerical solution was obtained by a finite element discretization so that there are no restrictions in the cross-sectional geometry or the distribution of material properties. The solution allows for arbitrary definition of strain measures using finite section modes and can consider the local effect of the longitudinal variation of strains to account, for instance, for end-effects due to constrained warping or for a Vlasov-like mode of deformation. As a result, the present methodology, implemented in the computer code UM/VABS, provides a solution to virtually all typical problems in the generation of one-dimensional models of (active) structures: analysis of solid and thin-walled beams, prismatic and initially curved reference lines at arbitrary locations, nonclassical (higher-order) deformations and end effects, and hygrothermal and electric interactions.

Numerical examples illustrated the use of the formulation for the analysis of both solid and thin-walled active beams. The proposed cross-sectional analysis defines elastic constants that fully capture the strain energy per unit length of the deformed structure as shown by comparisons with three-dimensional finite element solutions. The formulation also provides accurate recovery relations of the elastic and electric fields in the original three-dimensional domain, which can estimate the internal stress and electric field distributions in the structure and/or define the complete wet surface of a fluid-structure interaction problem. The formulation also introduced a simple yet effective approximation to the transverse shear effects using finite section modes. Moreover, higher-order deformations within the context of a purely one-dimensional theory were exemplified through the modeling of platelike deformations in an active thin-walled construction. As shown in this paper, the resulting model is several orders of magnitude smaller than a three-dimensional finite-element representation of the structure with comparable accuracy. Obtaining one-dimensional models that adequately represent the actual threedimensional state is particularly decisive in the analysis of slender structures undergoing large deflections, where the nonlinear effects are mainly associated with the longitudinal dimension of the structure.

Regarding models of active slender structures, the direct solution of the two-field electroelastic problem was conducted. The numerical results showed the importance of the piezoelectric coupling and electrode/actuator geometry in the actual cross-sectional characteristics and how they deviate from the typical prescribed field solution. The elastic stiffening of a cross section due to the energy stored in the electric field was shown to be negligible for typical piezoelectric material constants. The same behavior was observed in the cross-sectional capacitance, which showed negligible electric stiffening due to the energy stored in the induced elastic deformations. It was also shown, however, that the geometric properties of the cross section have a significant effect in the final actuation forces and capacitance per unit length. Therefore, for proper estimation of the actuation forces for generic electrode/actuator geometric arrangements, a two-field coupled theory with a detailed spatial discretization should be used, as described in this paper.

\section{Appendix: Submatrices in the FEM Discretization of the Cross-Sectional Electric Enthalpy}

The discrete form of the enthalpy per unit length, or crosssectional enthalpy, was presented in Eq. (25) as a function of the discrete cross-sectional state variable $z$ and the distribution of temperatures $T$. The coefficient matrices $A_{n}$ and vectors $\boldsymbol{b}_{n}$ in that expression are given here.

To simplify the resulting expressions, an equivalent state variable $z^{e}$ can be introduced as

$$
z^{e}=\left\{\epsilon^{T} \quad W^{T} \quad \Phi^{T} \quad W^{\prime} \quad \Phi^{\prime T}\right\}^{T}, \quad z^{e}=\Lambda \cdot z
$$

Then, the coefficient matrices and vectors in the cross-sectional enthalpy corresponding to the original state variable $z$ can be easily rewritten as a function of those of the new state variable, as follows:

$$
A_{n}=\Lambda^{T} A_{n}^{e} \Lambda, \quad \boldsymbol{b}_{n}=\Lambda^{T} b_{n}^{e}
$$

The submatrices in the problem are

$$
\begin{gathered}
A_{0}^{e}=\left[\begin{array}{ccccc}
0 & 0 & 0 & 0 & 0 \\
0 & D_{h h} & D_{h \phi} & 0 & 0 \\
0 & D_{\phi h} & D_{\phi \phi} & 0 & 0 \\
0 & 0 & 0 & 0 & 0 \\
0 & 0 & 0 & 0 & 0
\end{array}\right] \\
A_{1}^{e}=\left[\begin{array}{ccccc}
0 & D_{\epsilon h} & D_{\epsilon \phi} & 0 & 0 \\
D_{h \epsilon} & 2 D_{h R s} & D_{R \phi} & D_{h l} & D_{h \phi^{\prime}} \\
D_{\phi \epsilon} & D_{\phi R} & 0 & D_{\phi l} & D_{\phi \phi^{\prime}} \\
0 & D_{l h} & D_{l \phi} & 0 & 0 \\
0 & D_{\phi^{\prime} h} & D_{\phi^{\prime} \phi} & 0 & 0
\end{array}\right] \\
A_{2}^{e}=\left[\begin{array}{ccccc}
D_{\epsilon \epsilon} & D_{\epsilon R} & 0 & D_{\epsilon l} & D_{\epsilon \phi^{\prime}} \\
D_{R \epsilon} & D_{R R} & 0 & D_{R l} & D_{R \phi^{\prime}} \\
0 & 0 & 0 & 0 & 0 \\
D_{l \epsilon} & D_{l R} & 0 & D_{l l} & D_{l \phi^{\prime}} \\
D_{\phi^{\prime} \epsilon} & D_{\phi^{\prime} R} & 0 & D_{\phi^{\prime} l} & D_{\phi^{\prime} \phi^{\prime}}
\end{array}\right]
\end{gathered}
$$




$$
b_{1}^{e}=\left\{\begin{array}{c}
0 \\
D_{h T} \\
0 \\
0 \\
0
\end{array}\right\}, \quad b_{2}^{e}=\left\{\begin{array}{c}
D_{\epsilon T} \\
D_{R T} \\
0 \\
D_{l T} \\
0
\end{array}\right\}
$$

with

$$
\begin{gathered}
D_{\epsilon \epsilon}=\left\langle\Gamma_{\epsilon}^{T} c^{E} \Gamma_{\epsilon}\right\rangle, \quad D_{h R}=\left\langle\left(\Gamma_{h} N_{w}\right)^{T} c^{E} \Gamma_{R} N_{w}\right\rangle \\
D_{\epsilon T}=-\left\langle\Gamma_{\epsilon}^{T} c^{E} \alpha N_{T}\right\rangle \\
D_{h h}=\left\langle\left(\Gamma_{h} N_{w}\right)^{T} c^{E} \Gamma_{h} N_{w}\right\rangle, \quad D_{h l}=\left\langle\left(\Gamma_{h} N_{w}\right)^{T} c^{E} \Gamma_{l} N_{w}\right\rangle \\
D_{h T}=-\left\langle\left(\Gamma_{h} N_{w}\right)^{T} c^{E} \alpha N_{T}\right\rangle \\
D_{R R}=\left\langle\left(\Gamma_{R} N_{w}\right)^{T} c^{E} \Gamma_{R} N_{w}\right\rangle, \quad D_{R \epsilon}=\left\langle\left(\Gamma_{R} N_{w}\right)^{T} c^{E} \Gamma_{\epsilon}\right\rangle \\
D_{R T}=-\left\langle\left(\Gamma_{R} N_{w}\right)^{T} c^{E} \alpha N_{T}\right\rangle \\
D_{l l}=\left\langle\left(\Gamma_{l} N_{w}\right)^{T} c^{E} \Gamma_{l} N_{w}\right\rangle, \quad D_{R l}=\left\langle\left(\Gamma_{R} N_{w}\right)^{T} c^{E} \Gamma_{l} N_{w}\right\rangle \\
D_{l T}=-\left\langle\left(\Gamma_{l} N_{w}\right)^{T} c^{E} \alpha N_{T}\right\rangle \\
D_{h \epsilon}=\left\langle\left(\Gamma_{h} N_{w}\right)^{T} c^{E} \Gamma_{\epsilon}\right\rangle, \quad D_{l \epsilon}=\left\langle\left(\Gamma_{l} N_{w}\right)^{T} c^{E} \Gamma_{\epsilon}\right\rangle \quad(\mathrm{A} 4) \\
D_{\phi \phi}=-\left\langle\left(\nabla_{\xi} N_{\phi}\right)^{T} \varepsilon_{t}^{\Gamma} \nabla_{\xi} N_{\phi}\right\rangle, \quad D_{\phi^{\prime} \phi^{\prime}}=-\left\langle N_{\phi}^{T} \varepsilon_{l}^{\Gamma} N_{\phi}\right\rangle \\
D_{\phi \phi^{\prime}}=-\left\langle\left(\nabla_{\xi} N_{\phi}\right)^{T} \varepsilon_{t l}^{\Gamma} N_{\phi}\right\rangle \\
D_{\epsilon \phi}=\left\langle\Gamma_{\epsilon}^{T} e_{t}^{T} \nabla_{\xi} N_{\phi}\right\rangle, \quad D_{\epsilon \phi^{\prime}}=\left\langle\Gamma_{\epsilon}^{T} e_{l}^{T} N_{\phi}\right\rangle \\
D_{h \phi}=\left\langle\left(\Gamma_{h} N_{w}\right)^{T} e_{t}^{T} \nabla_{\xi} N_{\phi}\right\rangle, \quad D_{h \phi^{\prime}}=\left\langle\left(\Gamma_{h} N_{w}\right)^{T} e_{l}^{T} N_{\phi}\right\rangle \\
D_{R \phi}=\left\langle\left(\Gamma_{R} N_{w}\right)^{T} e_{t}^{T} \nabla_{\xi} N_{\phi}\right\rangle, \quad D_{R \phi^{\prime}}=\left\langle\left(\Gamma_{R} N_{w}\right)^{T} e_{l}^{T} N_{\phi}\right\rangle \\
D_{l \phi}=\left\langle\left(\Gamma_{l} N_{w}\right)^{T} e_{t}^{T} \nabla_{\xi} N_{\phi}\right\rangle, \quad D_{l \phi^{\prime}}=\left\langle\left(\Gamma_{l} N_{w}\right)^{T} e_{l}^{T} N_{\phi}\right\rangle \quad(\mathrm{A} S)
\end{gathered}
$$

Subindexes $l$ and $t$ in the matrices of piezoelectric constants refer to the longitudinal and transversal components, respectively. In particular, $e_{l}=\left[e_{1 i j}\right], e_{t}=\left[e_{\alpha i j}\right]$.

\section{Acknowledgments}

This work was sponsored by the Army Research Office under Grant 43854-EG. The Technical Monitor is Gary L. Anderson.

\section{References}

${ }^{1}$ Wada, B. K., Fanson, J. I., and Crawley, E. F., "Adaptive Structures," Mechanical Engineering, Vol. 112, No. 11, 1990, pp. 41-46.

${ }^{2}$ Tiersten, H. F., "On the Nonlinear Equations of Thermoelectroelasticity," International Journal of Engineering Sciences, Vol. 9, No. 7, 1971, pp. $587-604$.

${ }^{3}$ Chopra, I., "Review of Current Status of Smart Structures and Integrated Systems," AIAA Journal, Vol. 40, No. 11, 2002, pp. 2145-2187.

${ }^{4}$ Bernhard, A. P. F., and Chopra, I., "Analysis of a Bending-Torsion Coupled Actuator for a Smart Rotor with Active Blade Tips," Smart Materials and Structures, Vol. 10, No. 1, 2001, pp. 35-52.

${ }^{5}$ Cesnik, C. E. S., and Shin, S. J., "On the Modeling of Integrally Actuated Helicopter Blades," International Journal of Solids and Structures, Vol. 38, No. 10-13, 2001, pp. 1765-1789.

${ }^{6}$ Park, C. H., "On the Circuit Model of Piezoceramics," Journal of Intelligent Material Systems and Structures, Vol. 12, No. 7, 2001, pp. 515-522.

${ }^{7}$ Hagood, N. W., Chung, W. H., and Flotow, A., "Modelling of Piezoelectric Actuator Dynamics for Active Structural Control," Proceedings of the 31st AIAA/ASME/ASCE/AHS/ASC Structures, Structural Dynamics, and Materials Conference, AIAA, Washington, DC, 1990, pp. 2242-2256.

${ }^{8}$ Ahmad, S. N., Upadhyay, C. S., and Venkatesan, C., "Linear and Nonlinear Analysis of a Smart Beam Using a General Electrothermoelastic Formulation," AIAA Journal, Vol. 42, No. 4, 2004, pp. 840-849.

${ }^{9}$ Bent, A. A., "Active Fiber Composite Material Systems for Structural
Control Applications," Proceedings of the 1999 SPIE Symposium on Smart Structures and Materials, Society for Photo-Optical Instrumentation Engineers (International Society for Optical Engineering), Bellingham, WA, 1999, pp. 166-177.

${ }^{10}$ Wilkie, W. K., Bryant, R. G., High, J. W., Fox, R. L., Hellbaum, R. F., Jalink, A., Little, B. D., and Mirick, P. H., "Low-Cost Piezocomposite Actuator for Structural Control Applications," Proceedings of the 2000 SPIE Symposium on Smart Structures and Materials, Society for Photo-Optical Instrumentation Engineers (International Society for Optical Engineering), Bellingham, WA, pp. 323-334.

${ }^{11}$ Volovoi, V. V., Hodges, D. H., Cesnik, C. E. S., and Popescu, B., "Assessment of Beam Modeling Methods for Rotor Blade Applications," Mathematical and Computer Modelling, Vol. 33, No. 11, 2001, pp. 1099-1112.

${ }^{12}$ Jung, S. N., Nagaraj, V. T., and Chopra, I., "Assessment of Composite Rotor Blade Modeling Techniques," Journal of the American Helicopter Society, Vol. 44, No. 3, pp. 188-205.

${ }^{13}$ Berdichevsky, V. L., "On the Energy of an Elastic Rod," PMM, Journal of Applied Mathematics and Mechanics, Vol. 45, No. 4, 1982, pp. 518-529.

${ }^{14}$ Karwowski, A. J., "Asymptotic Models for a Long, Elastic Cylinder," Journal of Elasticity, Vol. 24, No. 1-3, 1990, pp. 229-287.

${ }^{15}$ Cesnik, C. E. S., and Hodges, D. H., "VABS: A New Concept for Composite Rotor Blade Cross-Sectional Modeling," Journal of the American Helicopter Society, Vol. 42, No. 1, 1997, pp. 27-38.

${ }^{16}$ Parker, D. F., "An Asymptotic Analysis of Large Deflections and Rotations of Elastic Rods," International Journal of Solids and Structures, Vol. 15, No. 5, 1979, pp. 361-377.

${ }^{17}$ Fan, H., and Widera, G. E. O., "Refined Engineering Beam Theory Based on the Asymptotic Expansion Approach," AIAA Journal, Vol. 29, No. 1, 1990, pp. 444-449.

${ }^{18}$ Kolpakov, A. G., "The Asymptotic Theory of Thermoelastic Beams," Journal of Applied Mechanics and Technical Physics, Vol. 36, No. 5, 1995, pp. 756-763.

${ }^{19}$ Buannic, N., and Cartraud, P., "Higher-Order Effective Modeling of Periodic Heterogeneous Beams. I: Asymptotic Expansion Method," International Journal of Solids and Structures, Vol. 38, No. 40-41, 2001, pp. 7139-7161.

${ }^{20}$ Berdichevsky, V. L., "Variational-Asymptotic Method of Constructing a Theory of Shells," PMM, Journal of Applied Mathematics and Mechanics, Vol. 43, No. 4, 1982, pp. 664-687.

${ }^{21}$ Hodges, D. H., Atilgan, A. R., Cesnik, C. E. S., and Fulton, M. V., "On a Simplified Strain Energy Function for Geometrically Nonlinear Behaviour of Anisotropic Beams," Composites Engineering, Vol. 2, No. 5-7, 1992, pp. 513-526.

${ }^{22}$ Cesnik, C. E. S., Hodges, D. H., and Sutyrin, V. G., "Cross-Sectional Analysis of Composite Beams Including Large Initial Twist and Curvature Effects," AIAA Journal, Vol. 34, No. 9, 1996, pp. 1913-1920

${ }^{23}$ Cesnik, C. E. S., Sutyrin, V. G., and Hodges, D. H., "Refined Theory of Composite Beams: The Role of Short-Wavelength Extrapolation," International Journal of Solids and Structures, Vol. 33, No. 10, 1996, pp. 1387-1408.

${ }^{24}$ Popescu, B., Hodges, D. H., and Cesnik, C. E. S., "Obliqueness Effects in Asymptotic Cross-Sectional Analysis of Composite Beams," Computers and Structures, Vol. 76, No. 4, 2000, pp. 533-543.

${ }^{25}$ Popescu, B., and Hodges, D. H., "On Asymptotically Correct Timoshenko-like Anisotropic Beam Theory," International Journal of Solids and Structures, Vol. 37, No. 3, 2000, pp. 535-558.

${ }^{26}$ Yu, W., Hodges, D. H., and Cesnik, C. E. S., "On Timoshenko-Like Modeling of Initially Curved and Twisted Composite Beams," International Journal of Solids and Structures, Vol. 39, No. 19, 2002, pp. 5101-5121.

${ }^{27}$ Cesnik, C. E. S., and Ortega-Morales, M., "Active Beam CrossSectional Modeling," Journal of Intelligent Material Systems and Structures, Vol. 12, No. 7, 2001, pp. 483-496.

${ }^{28}$ Danielson, D. A., and Hodges, D. H, "Nonlinear Beam Kinematics by Decomposition of the Rotation Tensor," Journal of Applied Mechanics, Vol. 54, No. 2, 1987, pp. 258-262.

${ }^{29}$ IEEE Std 176-1987, IEEE Standard on Piezoelectricity, Inst. of Electrical and Electronics Engineers, New York, 1988.

${ }^{30}$ Timoshenko, S. P., and Goodier, J. N., Theory of Elasticity, 3rd ed., McGraw-Hill, Maiden-Head, England, U.K., 1970.

${ }^{31}$ Gruttmann, F., and Wagner, W., "Shear Correction Factors in Timoshenko's Beam Theory for Arbitrary Shaped Cross-Sections," Computational Mechanics, Vol. 27, No. 3, 2001, pp. 199-207.

${ }^{32}$ Librescu, L., Meirovitch, L., and Na, S. S., "Control of Cantilever Vibration via Structural Tailoring and Adaptive Materials," AIAA Journal, Vol. 35, No. 8, 1997, pp. 1309-1315.

${ }^{33}$ Palacios, R., and Cesnik, C. E. S., "Structural Dynamics of Integrally Strained Slender Wings," Proceedings of the 2003 International Forum of Aeroelasticity and Structural Dynamics [CD-ROM], Confederation of European Aerospace Societies, Amsterdam, 2003, US-08.

B. Balachandran Associate Editor 